\title{
Hierarchical Control of Cognitive Processes: Switching Tasks in Sequences
}

\author{
Darryl W. Schneider and Gordon D. Logan \\ Vanderbilt University
}

\begin{abstract}
Hierarchical control of cognitive processes was studied by examining the relationship between sequenceand task-level processing in the performance of explicit, memorized task sequences. In 4 experiments, switch costs in task-switching performance were perturbed by sequence initiation times that varied with sequence complexity, preparation time, and type of sequence transition (repetition or switch). Hierarchical control was inferred from these sequence initiation time effects and the recurrent finding of no switch cost at the first serial position across sequences, the point at which sequence-level processes are likely active in maintaining or instantiating a hierarchical control structure in working memory. These findings resonate with past research on motor programs and serial memory and provide new insights into the concepts of task set and control.
\end{abstract}

Keywords: control, hierarchy, plan, sequence, task switching

Many of the tasks performed in everyday life are organized in sequences. For example, cooking food by following a recipe involves switching between different tasks (e.g., peeling, chopping, and measuring) in a prescribed order. Some tasks may be performed repeatedly (e.g., chopping), whereas other tasks may be performed only once (e.g., measuring), but all of the tasks are directed toward the fulfillment of the goal of producing edible (and hopefully tasty) food. Each task can be interpreted as a sequence of subtasks requiring specific motor actions (e.g., grasping, lifting, and pouring). Different tasks may involve some of the same subtasks, but in different sequences. To a naive observer, cooking might be perceived as a chain of motor actions that lacks structure, but to an informed observer, the motor actions occur in sequences corresponding to tasks that are organized according to the recipe.

A recipe is an example of a plan for behavior, which can be defined as "any hierarchical process in the organism that can control the order in which a sequence of operations is to be performed" (Miller, Galanter, \& Pribram, 1960, p. 16). Three concepts from this definition deserve emphasis: hierarchical, control, and sequence. Plans such as recipes are hierarchical in that tasks are composed of subtasks, which in turn are composed of specific motor actions. Plans control operations such as cognitive and motor processes because they indicate whether and when these operations are to be performed, enabling the selection of relevant processes and solving the problem of serial order in behavior (Lashley, 1951). This control extends to a sequence of operations because we are often concerned with behavior involving the coordination of multiple processes, even if those processes are not directly related to each other or to an overarching goal.

Darryl W. Schneider and Gordon D. Logan, Department of Psychology, Vanderbilt University.

This research was supported by National Science Foundation Grants BCS 0133202 and BCS 0446806 to Gordon D. Logan. We thank Bernhard Hommel, Mike Kane, Iring Koch, and David Rosenbaum for comments on this research.

Correspondence concerning this article should be addressed to Darryl W. Schneider, Department of Psychology, Vanderbilt University, Nashville, TN 37203. E-mail: darryl.schneider@vanderbilt.edu
Plans are studied under the rubric of control in many areas of cognitive psychology, but the control embodied by a plan is different from other conceptualizations of control. For example, much research is currently focused on executive control-how the mind controls itself. Executive control can be defined as the output of high-level processes involved in setting goals, selecting strategies to meet goals, programming low-level processes to implement strategies, and monitoring performance (Logan, 1985, 2003; Logan \& Gordon, 2001; Monsell, 1996; Monsell \& Driver, 2000b; Norman \& Shallice, 1986). This process-oriented approach to control is important for understanding cognition, but it is equally important to consider the representations that structure control. Our goal in the present study was to explore the control that arises from the hierarchical relationship between different levels of a simple plan: hierarchical control.

\section{Task Sequences and Hierarchical Control Structures}

We approached the issue of hierarchical control by investigating the performance of task sequences. In the present study, a task sequence was an arbitrary ordering of multiple occurrences of two tasks, without any relation to an overarching goal. Subjects were simply given a list of unrelated tasks and asked to perform the task sequence iteratively (cf. Logan, 2004). We argue that such task sequences can be encoded as plans, but more specifically as hierarchical control structures that guide individual task performance (De Jong, 1995; Lien \& Ruthruff, 2004; see also Luria \& Meiran, 2003).

We assume that a hierarchical control structure is an organized representation of control elements (Rosenbaum, Kenny, \& Derr, 1983; see also Rosenbaum, Inhoff, \& Gordon, 1984) with task identity and serial position codes, which is consistent with proposed representations of serially ordered items in memory (e.g., Anderson, Bothell, Lebiere, \& Matessa, 1998; Anderson \& Matessa, 1997) and tasks in dual-task performance (Logan \& Gordon, 2001; Luria \& Meiran, 2003). This structure is essential for accurate performance if one considers the following sequences of two tasks (denoted A and B) used in the present study: AABB and ABBA. Each task occurs with the same frequency in each se- 
quence, but not always in the same serial position. If the only information about which task to perform on a given trial is represented in the sequence (i.e., there are no external task cues), then the identity and serial position of each task must be coded in working memory (Logan, 2004).

Hierarchical control is manifest in the relationship between the sequence level (the representation of the task sequence and the processes that act on it) and the task level (the representations of individual tasks and their component processes). The representation of the task sequence need not be hierarchical (i.e., organized into discrete chunks), and the representations of the individual tasks need not be hierarchical (cf. Kleinsorge \& Heuer, 1999; Kleinsorge, Heuer, \& Schmidtke, 2004). What is important is the relationship between the sequence and task levels: What happens at the sequence level determines what happens at the task level. The relationship may be relatively straightforward: The task that is coded at the relevant serial position in the task sequence determines which task is performed. The relationship may also be more complex: Sequence-level processing may alter task-level processing because the representations and processes for the task sequence and individual tasks are assumed to share working memory capacity. Structurally, a hierarchical control structure is an organized representation of control elements, but functionally, it is a relationship between different levels of representation and processing.

Although our definition of a hierarchical control structure was derived from the motor programming and serial memory literatures, there is an important distinction between past research and the present study. Hierarchical control structures are concerned with the organization of motor actions in the motor programming literature (Rosenbaum et al., 1983) and with the organization of stimuli in the serial memory literature (Anderson \& Matessa, 1997). Hierarchical control structures in the present study are concerned with the organization of tasks or task goals. These tasks may involve the processing of many different stimuli and the execution of a variety of motor actions, but the organization of behavior takes place at a more abstract level (see also Cooper \& Shallice, 2000, in press). In this sense, the present study is a conceptual amalgamation of past research, with the organization of stimuli (i.e., task identities) at the sequence level controlling the organization of motor actions (i.e., components of task performance) at the task level.

A hierarchical control structure can be induced in working memory by manipulating stimulus properties or experimental instructions. Lien and Ruthruff (2004) demonstrated that manipulating the spatial and temporal grouping of stimuli could induce a hierarchical control structure. In most situations, however, hierarchical control structures arise from experimental instructions. For example, the order in which tasks are to be performed in dual-task performance is often explicitly indicated in the instructions (e.g., Pashler, 1984; but see De Jong, 1995). We assume that experimental instructions are translated into a propositional representation of a task sequence that organizes and specifies the elements of a hierarchical control structure (Logan \& Gordon, 2001; for related work in a different context, see Dixon, 1987).

The hierarchical control structure for a task sequence fits the earlier definition of a plan (Miller et al., 1960). Individual tasks are performed in accordance with the task sequence by accessing relevant information in the hierarchical control structure, resulting in organized behavior. The distinction between individual tasks and task sequences brings attention to the importance of investi- gating the relationship between the sequence and task levels and the implications of this relationship for understanding control. The levels may be independent, with sequence-level processing having no effect on task-level processing. However, the levels may interact, with sequence-level processing affecting task-level processing, or vice versa. In the present study, we addressed this issue by examining how task transitions (repeating and switching tasks) are affected by sequence transitions (repeating and switching sequences).

\section{Switching Tasks in Sequences}

A hierarchical control structure provides a way of coordinating cognitive processes for switching between the performance of different tasks. This kind of switching has been studied extensively in experiments on task switching (Jersild, 1927; Logan, 2003; Mayr, 2003; Monsell, 2003). People are slower and less accurate when switching than when repeating tasks, and this switch cost has been attributed to a variety of processes.

Some authors interpret switch costs as indices of timeconsuming executive control processes involved in task-set reconfiguration-changing the state of the cognitive system when switching tasks (e.g., Meiran, 1996; Rogers \& Monsell, 1995). Other authors interpret switch costs as priming or interference effects arising from past cues, stimuli, or task sets in memory (e.g., Allport, Styles, \& Hsieh, 1994; Allport \& Wylie, 2000; Schneider \& Logan, 2005; Waszak, Hommel, \& Allport, 2003). These interpretations are not mutually exclusive, and many authors endorse aspects of both of them (e.g., Meiran, Chorev, \& Sapir, 2000). Although the hypothesized processes underlying switch costs differ in many respects, they share a common locus of activity: individual tasks. The present study is concerned with processes that function at a higher level (i.e., task sequences) and how their activity affects task-level processes. We chose to examine how switch costs at the task level are affected by transitions at the sequence level.

Some of the extant research on switching tasks in sequences suggests a lack of a functional relationship between the sequence and task levels. Logan (2004) found that the probability of performing a task sequence perfectly from memory was unaffected by the number of task switches in the sequence. Explicit knowledge of a task sequence or foreknowledge of task transitions does not decrease switch cost relative to a lack of such knowledge (Kleinsorge, Schmidtke, Gajewski, \& Heuer, 2003; Sohn \& Carlson, 2000; but see Sohn \& Anderson, 2001). Expected task transitions do not decrease switch cost relative to unexpected task transitions (Ruthruff, Remington, \& Johnston, 2001; see also Dreisbach, Haider, \& Kluwe, 2002). A predictable task sequence that is learned implicitly does not lead to a smaller switch cost than a novel task sequence (Gotler, Meiran, \& Tzelgov, 2003; Heuer, Schmidtke, \& Kleinsorge, 2001; Koch, 2001, 2005). Finally, many researchers have argued that a predictable task sequence should decrease switch cost when there is ample time to prepare for a task switch (e.g., Rogers \& Monsell, 1995), but manipulations of preparation time have yielded mixed results (Altmann, 2004).

Other studies on manipulating task order in dual-task performance and switching tasks in sequences have provided some evidence for a functional relationship between the sequence and task levels. Subjects tend to repeat task order in dual-task performance and prepare for rapid switches from the first to the second 
task when task order alternates (De Jong, 1995); the latter result is supported by the finding that switching task order leads to an order switch cost that decreases as preparation time increases (Luria \& Meiran, 2003). These authors suggested that "the sequential preparation for and performance of the individual tasks in overlappingtask performance is coordinated and controlled by a multi-level control structure" (De Jong, 1995, p. 21) and that subjects "activate an explicit representation of the subtask sequence during online order control" (Luria \& Meiran, 2003, p. 563).

Lien and Ruthruff (2004) manipulated the spatial and temporal grouping of stimuli to induce a hierarchical control structure when switching tasks in sequences, discovering that stronger manipulations led to increasingly smaller relative switch costs. ${ }^{1}$ In some cases, they obtained no switch costs at all and proposed that preparing for a task sequence can eliminate task-level effects. Koch, Philipp, and Gade (2006) found that task-set inhibition (slower performance for $n-2$ task repetitions than for $n-2$ task switches; see Mayr \& Keele, 2000) was attenuated when the $n-2$ task repetitions occurred within an explicitly known task sequence, which was interpreted as evidence that the hierarchical structure of the task sequence facilitated task performance. In the task span procedure, in which subjects perform a memorized list of tasks, Logan (2004) obtained evidence of chunking with long task sequences (indicative of hierarchical organization in memory; see Bower \& Winzenz, 1969) and noted that subjects were slow when initiating a sequence, an effect that tended to increase with sequence length. Logan (in press) also found that switch costs were larger in the task span procedure than in explicit task-cuing procedures and were affected by keeping track of task order and protecting task-level performance from content-specific interference from a memorized sequence.

Evidence is starting to accumulate for a functional relationship between the sequence and task levels, but there are mixed results in the literature. A critical issue that has not been adequately addressed in many previous studies is what happens at sequence transitions, when sequence-level processes would presumably have to be active to instantiate or maintain a hierarchical control structure in working memory. The operation of these high-level processes for sequence transitions could change the state of the cognitive system and affect the low-level processes associated with task transitions, possibly perturbing switch costs. Identifying such an interaction would provide strong evidence that sequencelevel processing affects task-level processing, and represent a compelling example of hierarchical control in cognition.

\section{The Present Study}

We addressed the relationship between sequence- and task-level processing with four experiments in which subjects repeated and switched tasks that were organized in explicit, memorized sequences. All experiments were based on the following procedure: At the start of a block of trials, subjects memorized a task sequence involving task switches and task repetitions (e.g., ABBA). The sequence was then performed repeatedly during the block (except in Experiment 4) on a series of target stimuli. Except for the requirement to repeat the sequence, this procedure resembles the task span procedure developed by Logan $(2004,2006)$ to investigate the relationship among working memory, task switching, and executive control. An advantage of this procedure is that it allows us to have greater confidence in attributing any changes in switch cost to hierarchical control from the sequence level to the task level (rather than to other effects, such as priming of cue encoding; e.g., Schneider \& Logan, 2005), because subjects must rely on their memory of the task sequence to perform the correct task on each trial (see also Koch, 2003). If subjects forget the task sequence, they will not know which task to perform, because there are no external task cues.

To assess potential interactions between sequence- and tasklevel processing, we calculated two measures of switch cost in our analyses: overall and within-sequence switch costs. We define overall switch cost as the difference between task switches and task repetitions, irrespective of where those task transitions occur in a sequence. This is the standard measure of switch cost in almost all task-switching studies, but it will become clear from our results that it is a flawed measure if one does not recognize the contribution of hierarchical control to task performance. We define withinsequence switch cost as the difference between task switches and task repetitions, excluding the first serial position in a sequence. The rationale for calculating within-sequence switch cost is that if there is a relationship between sequence- and task-level processing, it should be most pronounced on trials that mark sequence transitions (i.e., the first serial position of a sequence). We are not excluding the possibility that sequence-level processing can affect task performance beyond the first serial position, but such effects are likely to be weaker.

If task-level processing is unaffected by sequence-level processing, then there are two basic predictions in our experiments. First, overall switch costs (as well as within-sequence switch costs) should not differ between different sequences, especially when the sequences have the same task and task transition frequencies and produce identical patterns of task transitions across an extended series of trials (e.g., AABB and ABBA sequences). Second, switch costs should not be affected at sequence transitions, regardless of the type of sequence-level processing (e.g., repeating or switching sequences) that is required to identify the relevant task.

If task-level processing is affected by sequence-level processing, then overall switch costs (but not necessarily within-sequence switch costs) should differ between sequences. Moreover, switch costs should be affected at sequence transitions, becoming either larger or smaller owing to sequence-level processing. This latter effect would provide strong evidence of hierarchical control because it would imply a functional relationship between the sequence and task levels, with task-level effects being altered by sequence-level processing.

\section{Experiment 1}

The purpose of Experiment 1 was to obtain evidence of hierarchical control between the sequence and task levels. Subjects

\footnotetext{
${ }^{1}$ In Lien and Ruthruff's (2004) experiments, relative switch cost was defined as the difference between task switches that were sequence repetitions and task repetitions that were sequence switches. Positive values were interpreted as evidence that the task level dominated the sequence level; negative values were associated with the reverse relationship. The validity of this measure rests on the assumption that processing at one level has no effect on processing at the other level. Our data and those of Lien and Ruthruff (2004, Experiments 6 and 7) do not support this assumption; therefore, we adopted different measures of switch cost in the present study.
} 
performed $\mathrm{AABB}$ and ABBA task sequences. We calculated overall and within-sequence switch costs to gauge differences between the sequences. We expected differences in overall switch costs due to sequence initiation time effects-slower performance at the first serial position relative to subsequent serial positions, reflecting the time required to restart a sequence. This expectation was motivated by a number of findings.

First, hierarchical control in the performance of motor sequences is pronounced at the boundaries of organizational units (for a review, see Keele, Cohen, \& Ivry, 1990). The organizational unit in the present study is the task sequence; therefore, we expected hierarchical control to be manifest at sequence transitions. Note that these sequence transitions occur at an abstract level: A task sequence does not specify a sequence of motor actions but rather a sequence of task goals for what to do with a random series of target stimuli.

Second, there is evidence from Logan's (2004) task span procedure that performance is slower at the start of a sequence and that the degree of slowing is related to sequence length. However, in the task span procedure, the start of a sequence is always the first trial performed by subjects, so sequence initiation times are confounded with startup costs. This confound is not present in our experiments because subjects repeated every sequence several times and we excluded the first iteration of every sequence in our analyses.

Third, in Lien and Ruthruff's (2004) experiments on hierarchical control structures induced by stimulus factors, there was evidence of sequence initiation times with their two-task sequences (e.g., in the fixed-order condition in their Experiments 2-5). However, their sequence initiation times reflected spatial and temporal grouping of stimuli. We wanted to link sequence initiation to explicit knowledge of a task sequence in memory. ${ }^{2}$

Sequence initiation times and related effects such as restart costs have been observed in many studies (e.g., Allport \& Wylie, 2000; Anderson \& Matessa, 1997; Henry \& Rogers, 1960; Klapp, Anderson, \& Berrian, 1973; Logan, 2004; Povel \& Collard, 1982; Sternberg, Monsell, Knoll, \& Wright, 1978) and imply hierarchical control because task performance mirrors the structure of the sequence. A critical issue addressed in Experiment 1 is whether sequence initiation affects task-level performance beyond what we can infer from differences in overall switch costs.

If task-level processing depends on sequence-level processing, then switch costs should be affected at sequence transitions, as reflected by magnified or attenuated switch costs at the first serial position across sequences. Changes in switch cost across serial positions are related to but separate from sequence initiation time effects-it is possible to have one effect without the other. Obtaining altered switch costs at the first serial position, relative to within-sequence switch costs, would allow us to go beyond the inferences drawn from sequence initiation time effects and establish hierarchical control of the cognitive processes involved in task switching.

\section{Method}

Subjects. Twenty students from Vanderbilt University completed the experiment in exchange for course credit.

Apparatus and stimuli. The experiment was conducted using computers running E-Prime software (Version 1.1; Psychology Software Tools, 2002). Input was registered from standard keyboards, and output was displayed on monitors. Eight target stimuli were created from the factorial combination of three dimensions: color (red or blue), shape (circle or triangle), and size (large $[7.0 \times 7.0 \mathrm{~cm}]$ or small $[3.5 \times 3.5 \mathrm{~cm}])$. The two tasks were color and shape judgments. Target size was manipulated to increase the number of targets and reduce the number of identical target repetitions across trials. Viewing distance was unconstrained but approximately $60 \mathrm{~cm}$.

Procedure. Subjects performed the experiment in private testing rooms after giving informed consent. Instructions concerning the trial format, tasks, target stimuli, response-key mappings, and sequences were presented onscreen and explained by the experimenter. There were two sequences: $\mathrm{AABB}$ and $\mathrm{ABBA}$. Both sequences are matched for task frequency and task transition frequency, so any differences in switch cost cannot be attributed to differences in frequency. Across an extended series of trials, both sequences produce identical patterns of task transitions (e.g., ABBAABBA ...); the only difference is how trials are organized as iterations of a sequence. All possible designations of the color and shape tasks as A or B were used, yielding four task-specific sequences (CCSS, SSCC, CSSC, and SCCS, where C and S denote the color and shape tasks, respectively) that were displayed onscreen as uppercase words separated by commas (e.g., COLOR, COLOR, SHAPE, SHAPE for the CCSS sequence). Subjects were instructed to perform the tasks according to the task-specific sequence indicated at the start of each block of trials, repeating the sequence every four trials. The experimenter stressed the importance of remembering the relevant sequence during each block and responding quickly and accurately on every trial.

A block began with a screen indicating the relevant task-specific sequence, which had to be memorized because there was no reminder about the sequence during the block. Subjects completed two practice blocksone block for each sequence (AABB and ABBA), with the task-specific sequence randomly selected. Subjects then completed 20 experimental blocks-five blocks for each task-specific sequence, randomly selected with the restriction that each sequence be used once every four blocks. Blocks were separated by rest periods. There were 24 trials per block, allowing six iterations of the relevant sequence and three presentations of each target.

A trial began with the immediate onset of a randomly selected target. The target remained onscreen until the subject responded by pressing the $F$ or $J$ key. Response categories for the same task were assigned to different response keys, and the four possible response-key mappings were counterbalanced across subjects. Text indicating the response-key mappings appeared as a reminder for the duration of each trial in gray 14-point Courier New font in the bottom-left and bottom-right corners of the screen for the $F$ and $J$ keys, respectively. After a response, the target was erased and the next trial commenced after a response-stimulus interval of $500 \mathrm{~ms}$. The response time (RT) and response on each trial were recorded. The experiment lasted about 25 min.

\section{Results and Discussion}

Practice blocks and any blocks with error rates exceeding $20 \%$ ( $M=0.25$ blocks per subject) were excluded from all analyses. A block error rate criterion was implemented because a high error rate may reflect a failure to remember the relevant sequence during the block, obfuscating the interpretation of any effects. The first iteration of a sequence was excluded from all analyses to avoid any artifacts associated with block initiation (cf. Allport \& Wylie, 2000; Logan, 2004). Trials with RTs below $100 \mathrm{~ms}$ or above 4,000 $\mathrm{ms}(M=0.86 \%$ of trials per subject $)$ were excluded as outliers. Trials with errors were excluded from the RT analysis.

\footnotetext{
${ }^{2}$ We also wanted to demonstrate that a supposedly flat task structure (one that lacks spatial and temporal grouping; Lien \& Ruthruff, 2004) could be used to provide evidence of hierarchical control.
} 
We collapsed across task-specific sequences for the AABB and ABBA sequences (i.e., CCSS and SSCC; CSSC and SCCS) because they had identical patterns of data. Trials were classified as task switches or task repetitions according to the relationship between the tasks on trials $n$ and $n-1$. Mean RT and error rate were calculated across subjects for each serial position in each sequence. These data are provided in Table 1, and mean RTs are plotted in Figure 1. The RT and error rate data were submitted to separate 2 (sequence) $\times 4$ (serial position) repeated measures analyses of variance (ANOVAs), which are summarized in Table 2. The omnibus ANOVA effects are not of particular interest; therefore, planned comparisons using the error term from the Sequence $\times$ Serial Position interaction are reported below.

Error rate analysis. Mean error rate was $1.8 \%$ (see Table 1). Overall switch costs were $-0.1 \%$ and $1.4 \%$ for the AABB and ABBA sequences, respectively, which were significantly different, $F(1,57)=5.39, p<.05, \eta_{\mathrm{p}}^{2}=.09$. The difference in overall switch costs was primarily due to the absence of a switch cost when comparing the first serial position of each sequence: The first serial position of the AABB sequence was a task switch with $1.2 \%$ error rate, and the first serial position of the ABBA sequence was a task repetition with $1.2 \%$ error rate. Excluding the first serial position, within-sequence switch costs were $0.2 \%$ and $1.3 \%$ for the $\mathrm{AABB}$ and $\mathrm{ABBA}$ sequences, respectively, which were not significantly different, $F(1,57)=1.80, p=.19, \eta_{\mathrm{p}}^{2}=.03$. These data provide evidence of hierarchical control: sequence-level processing affected task-level processing at the first serial position of each sequence, producing no switch cost and perturbing overall switch cost. The nonsignificant difference in within-sequence switch costs suggests that these effects were attenuated beyond the first serial position.

RT analysis. Overall switch costs were $322 \mathrm{~ms}$ and $-45 \mathrm{~ms}$ for the $\mathrm{AABB}$ and $\mathrm{ABBA}$ sequences, respectively, which were significantly different, $F(1,57)=165.84, p<.001, \eta_{\mathrm{p}}^{2}=.74$. The difference in overall switch costs indicates that task-switching performance was affected by the different sequences. We examined the mean RTs across serial position for each sequence (see Figure 1), and the source of the difference was readily apparent: RT for the first serial position of each sequence (i.e., sequence initiation time) was much slower than the mean RT collapsed across subsequent serial positions, $F_{\mathrm{s}}(1,57)>213.64, p \mathrm{~s}<.001$, $\eta_{\mathrm{p}}^{2} \mathrm{~s}>$.78. Overall switch costs differed between sequences because sequence initiation times affected different task transitions. Repeating the sequence increased the overall switch cost for the

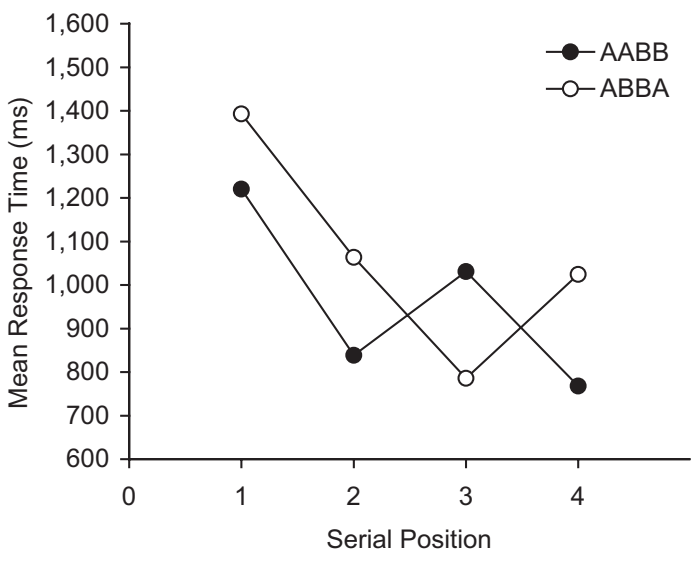

Figure 1. Mean response time for each serial position of each sequence in Experiment 1.

AABB sequence because the first serial position was a task switch but decreased the overall switch cost for the ABBA sequence because the first serial position was a task repetition (see Table 1).

A negative overall switch cost for the ABBA sequence does not mean that subjects were faster at switching than at repeating tasks. It is apparent in Table 1 that the task repetition occurring at the third serial position was much faster than either task switch in the sequence. Within-sequence switch costs were calculated to obtain a measure of task-switching performance that was relatively unaffected by sequence initiation. Within-sequence switch costs were $228 \mathrm{~ms}$ and $258 \mathrm{~ms}$ for the AABB and ABBA sequences, respectively, which were not significantly different, $F(1,57)<1$. These data are consistent with the error rate data in showing that the effects of hierarchical control were most pronounced for the first serial position and attenuated for subsequent serial positions. Moreover, these analyses suggest that overall switch cost may be a misleading measure of task switching when performing task sequences if one does not take into account the effects of hierarchical control.

Stronger evidence of hierarchical control comes from analysis of performance at the first serial position. RT at the first serial position of the AABB sequence (a task switch) was $173 \mathrm{~ms}$ faster than that of the ABBA sequence (a task repetition), $F(1,57)=$ $36.62, p<.001, \eta_{\mathrm{p}}^{2}=.39$, a "switch benefit" that was replicated in Experiments 3 and 4. The absence of a switch cost at the first

Table 1

Task Transition, Mean Response Time (RT; in Milliseconds), and Error Rate in Experiment 1

\begin{tabular}{lcccc}
\hline & \multicolumn{4}{c}{ Serial position } \\
\cline { 2 - 5 } $\begin{array}{c}\text { Sequence and } \\
\text { measure }\end{array}$ & 1 & 2 & 3 & 4 \\
\hline AABB & & & & \\
$\quad$ Task transition & TS & TR & TS & TR \\
RT & $1,220(47)$ & $839(31)$ & $1,031(42)$ & $768(29)$ \\
Error rate & $1.2(0.3)$ & $1.3(0.4)$ & $1.9(0.5)$ & $1.9(0.8)$ \\
ABBA & & & & TR \\
Task transition & TR & TS & $786(27)$ & $1,024(35)$ \\
RT & $1,393(45)$ & $1,064(40)$ & $1.5(0.4)$ & $2.2(0.6)$ \\
Error rate & $1.2(0.4)$ & $3.5(0.8)$ & & \\
\hline
\end{tabular}

Note. Standard errors appear in parentheses. TS $=$ task switch; TR $=$ task repetition. 
Table 2

Analyses of Variance on Mean Response Time and Error Rate in Experiment 1

\begin{tabular}{|c|c|c|c|c|c|c|c|}
\hline \multirow[b]{2}{*}{ Effect } & \multirow[b]{2}{*}{$d f \mathrm{~s}$} & \multicolumn{3}{|c|}{ Response time } & \multicolumn{3}{|c|}{ Error rate } \\
\hline & & $F$ & $M S E$ & $\eta_{\mathrm{p}}^{2}$ & $F$ & $M S E$ & $\eta_{\mathrm{p}}^{2}$ \\
\hline Sequence $(S)$ & 1,19 & $26.11^{* *}$ & $15,990.35$ & .58 & 2.96 & 3.59 & .14 \\
\hline Serial position $(\mathrm{P})$ & 3,57 & $105.65 * *$ & $14,461.95$ & .85 & 1.83 & 5.41 & .09 \\
\hline $\mathrm{S} \times \mathrm{P}$ & 3,57 & $67.26^{* *}$ & $8,159.07$ & .78 & $2.86^{*}$ & 4.34 & .13 \\
\hline
\end{tabular}

serial position replicates the error rate data and can be interpreted in many ways.

One interpretation is that whenever a sequence repeated, sequence-level processing was necessary to maintain the hierarchical control structure in working memory, either through rehearsal or by resetting the current position in the task sequence. Even though the hierarchical control structure remained unchanged, the act of maintaining it served to refresh or reset the contents of working memory, with at least two possible consequences. One possibility is that task sets are linked to the existing hierarchical control structure, and operating on that structure eliminates the activation of associated task sets. Some authors have suggested that switch costs are due to interference from previously activated task sets (e.g., Allport et al., 1994); therefore, eliminating such activation would eliminate switch costs. Another possibility is that when moving from the task level to the sequence level, the current task set may shift to a neutral state that is not biased toward one task over another. When performing the task at the first serial position of the sequence, the task set would then have to be "reconfigured" (Rogers \& Monsell, 1995) regardless of the task transition, yielding no switch cost.

We cannot distinguish between these possibilities with our data, but the overarching point is that they both reflect hierarchical control: sequence-level processing affected task-level processing in a way that resulted in no switch cost. This interpretation is consistent with the position of Lien and Ruthruff (2004), who argued that the benefit of a task repetition is fragile and can be eliminated in situations involving hierarchical control. However, contrary to Lien and Ruthruff's view, we obtained evidence of hierarchical control with a supposedly flat (i.e., nonhierarchical) task structure, suggesting that their perspective is too constrained. We argue that hierarchical control depends on the representational structure in working memory and is manifest in the relationship between the sequence and task levels: Sequence-level processing sets the stage for subsequent task-level processing, thereby perturbing task-level effects.

There is an alternative interpretation of the absence of a switch cost that does not imply hierarchical control. ${ }^{3}$ Sequence initiation and task switching may operate independently and in parallel. If sequence initiation takes longer than task switching and both types of processing must finish to enable task performance, then switch cost at the first serial position could be partially absorbed in the slack time produced by sequence initiation (Schweickert, 1978; Schweickert \& Townsend, 1989). For subsequent serial positions, sequence initiation would be unnecessary, resulting in no slack time and therefore no reduction in switch cost. This interpretation is consistent with our data but may not be plausible in practice. Even if sequence initiation and task switching were independent and could operate in parallel, it seems unlikely for task switching to occur while refreshing the representation of the task sequence in working memory from which the relevant task must be retrieved. ${ }^{4}$ If task performance depends on knowing the relevant task and knowing the relevant task depends on having the sequence readily available in memory, then task switching cannot begin while the sequence is still being processed in working memory. This interpretation becomes even more unlikely in the context of Experiment 4 , in which we obtained no switch cost at the first serial position when switching sequences. Task switching cannot occur in advance if the relevant task must be identified from a sequence that has yet to be instantiated in working memory. Consequently, we prefer to interpret the absence of a switch cost in the error rate and RT data as evidence of hierarchical control.

A remaining issue is why there was a switch benefit at the first serial position. We offer two possible reasons: (a) Sequence initiation may take longer when it involves a task repetition, implying that task-level processing affects sequence-level processing, or (b) sequence initiation may take longer when the structure of a sequence is more complex, implying that sequence-level processing is specific to the demands of the sequence. To elaborate on the latter reason, if we use the number of task switches within a sequence as an index of its complexity, the ABBA sequence involves two within-sequence task switches, whereas the AABB sequence involves one within-sequence task switch. If sequence initiation involves preparation for forthcoming task switches (Lien \& Ruthruff, 2004), then one would expect a slower sequence initiation time for the ABBA sequence compared with the AABB sequence, as we observed. Experiment 2 was designed to distinguish between these interpretations.

\section{Experiment 2}

Experiment 1 provided evidence of hierarchical control when switching tasks in sequences. The purpose of Experiment 2 was twofold: First, we wanted to extend the findings with four-task sequences in Experiment 1 to six-task sequences. Second, we wanted to examine differences in sequence initiation time for different sequences. Are sequence initiation times always slower for task repetitions than for task switches, reflecting "repetition costs" in task-switching performance? Alternatively, do the differ-

\footnotetext{
${ }^{3}$ We thank Bernhard Hommel for suggesting this interpretation.

${ }^{4}$ It may be possible to retain a variant of this interpretation by assuming that sequence- and task-level processes are cascaded, such that task-level processing can begin as soon as some information about the relevant task is available, even if sequence-level processing has not finished (McClelland, 1979).
} 
ences have nothing to do with task switching but instead reflect the sensitivity of sequence initiation to differences in sequence complexity?

Subjects performed AAABBB, AABABB, AABBBA, and $\mathrm{ABABBA}$ sequences. If sequence initiation times are slower for task repetitions than for task switches between iterations for sequences of comparable complexity, then first serial position RTs should be longer for the AABBBA and $\mathrm{ABABBA}$ sequences when compared with the $\mathrm{AAABBB}$ and $\mathrm{AABABB}$ sequences, respectively. If sequence initiation times vary as a function of sequence complexity, then a different pattern would be expected. For convenience, sequence complexity is indexed by the number of within-sequence task switches, a measure that correlates perfectly with other methods of coding the complexity of binary sequences (e.g., by successive run lengths; see Simon, 1972, p. 376). On the basis of this measure, first serial position RTs should be longer for the $\mathrm{AABABB}$ and $\mathrm{ABABBA}$ sequences (with three and four within-sequence task switches, respectively) when compared with the AAABBB and AABBBA sequences (with one and two withinsequence task switches, respectively), where the task transition between iterations is constant for each comparison. Differences in first serial position RTs for these comparisons would indicate that the process of sequence initiation is sensitive to sequence complexity and may involve preparation for the task transitions within the to-be-performed sequence.

\section{Method}

Subjects. Twenty students from Vanderbilt University completed the experiment in exchange for course credit. None of the subjects had taken part in Experiment 1.

Apparatus and stimuli. The apparatus and stimuli were identical to those in Experiment 1.

Procedure. The procedure was identical to that of Experiment 1, except for the following changes. There were four sequences: AAABBB, AABABB, AABBBA, and ABABBA. All sequences are matched for task frequency but not for task transition frequency. Sequences were chosen such that there were pairs of sequences (AAABBB and AABABB,
AABBBA and ABABBA) that were similar in structure and had the same task transition between iterations of each sequence. Note that the paired sequences differ only in the transposition of tasks at two serial positions (i.e., the $\mathrm{AAABBB}$ sequence becomes the $\mathrm{AABABB}$ sequence when the tasks at Serial Positions 3 and 4 are transposed; the AABBBA sequence becomes the ABABBA sequence when the tasks at Serial Positions 2 and 3 are transposed). All possible designations of the color and shape tasks as A or B were used, yielding eight task-specific sequences (CCCSSS, SSSCCC, CCSCSS, SSCSCC, CCSSSC, SSCCCS, CSCSSC, and SCSCCS). Subjects were instructed to perform the tasks according to the task-specific sequence indicated at the start of each block of trials, repeating the sequence every six trials.

Subjects completed two practice blocks-two of the four sequences (AAABBB, AABABB, AABBBA, and ABABBA) were randomly selected, with the task-specific sequence randomly selected. Subjects then completed 24 experimental blocks-three blocks for each task-specific sequence, randomly selected with the restriction that each sequence be used once every eight blocks. There were 48 trials per block, allowing eight iterations of the relevant sequence and six presentations of each target. The trial format was identical to that of Experiment 1. The experiment lasted about $45 \mathrm{~min}$.

\section{Results and Discussion}

Practice blocks and any blocks with error rates exceeding $20 \%$ ( $M=0.85$ blocks per subject) were excluded from all analyses. The first iteration of a sequence and trials with RTs below $100 \mathrm{~ms}$ or above $4,000 \mathrm{~ms}$ ( $M=1.17 \%$ of trials per subject $)$ were also excluded from all analyses. Trials with errors were excluded from the RT analysis.

We collapsed across task-specific sequences for the AAABBB, AABABB, AABBBA, and ABABBA sequences because they had identical patterns of data. Trials were classified as task switches or task repetitions, and then mean RT and error rate were calculated across subjects for each serial position in each sequence. These data are provided in Table 3, and mean RTs are plotted in Figure 2. The RT and error rate data were submitted to separate 4 (sequence) $\times 6$ (serial position) repeated measures ANOVAs, which are summarized in Table 4. The omnibus ANOVA effects

Table 3

Task Transition, Mean Response Time (RT; in Milliseconds), and Error Rate in Experiment 2

\begin{tabular}{|c|c|c|c|c|c|c|}
\hline \multirow{2}{*}{$\begin{array}{l}\text { Sequence and } \\
\text { measure }\end{array}$} & \multicolumn{6}{|c|}{ Serial position } \\
\hline & 1 & 2 & 3 & 4 & 5 & 6 \\
\hline \multicolumn{7}{|l|}{ AAABBB } \\
\hline Task transition & TS & TR & TR & TS & TR & TR \\
\hline RT & $1,121(61)$ & $703(33)$ & $634(29)$ & $958(55)$ & 657 (33) & $668(38)$ \\
\hline Error rate & $4.6(0.9)$ & $1.8(0.5)$ & $1.6(0.5)$ & $4.3(0.8)$ & $3.0(0.7)$ & $2.5(0.5)$ \\
\hline \multicolumn{7}{|l|}{ AABABB } \\
\hline Task transition & TS & TR & TS & TS & TS & TR \\
\hline RT & 1,431 (96) & $826(44)$ & $1,035(56)$ & $1,261(64)$ & $1,156(73)$ & 738 (33) \\
\hline Error rate & $3.3(0.7)$ & $1.5(0.6)$ & $4.2(0.9)$ & $3.9(0.6)$ & $4.7(0.8)$ & $2.1(0.5)$ \\
\hline \multicolumn{7}{|l|}{ AABBBA } \\
\hline Task transition & TR & TR & TS & TR & TR & TS \\
\hline RT & $1,045(101)$ & 747 (40) & $976(51)$ & $712(36)$ & $689(42)$ & 1,015 (49) \\
\hline Error rate & $2.0(0.6)$ & $3.6(1.2)$ & $4.2(0.8)$ & $2.1(0.6)$ & $3.0(0.9)$ & $4.0(0.8)$ \\
\hline \multicolumn{7}{|l|}{ ABABBA } \\
\hline Task transition & TR & TS & TS & TS & TR & TS \\
\hline RT & $1,421(83)$ & $1,004(45)$ & $1,208(46)$ & $1,165(52)$ & $887(50)$ & $1,055(46)$ \\
\hline Error rate & $2.1(0.5)$ & $5.5(1.1)$ & $4.3(0.8)$ & $4.3(0.7)$ & $2.5(0.8)$ & $4.2(1.0)$ \\
\hline
\end{tabular}

Note. Standard errors appear in parentheses. TS $=$ task switch; TR $=$ task repetition. 

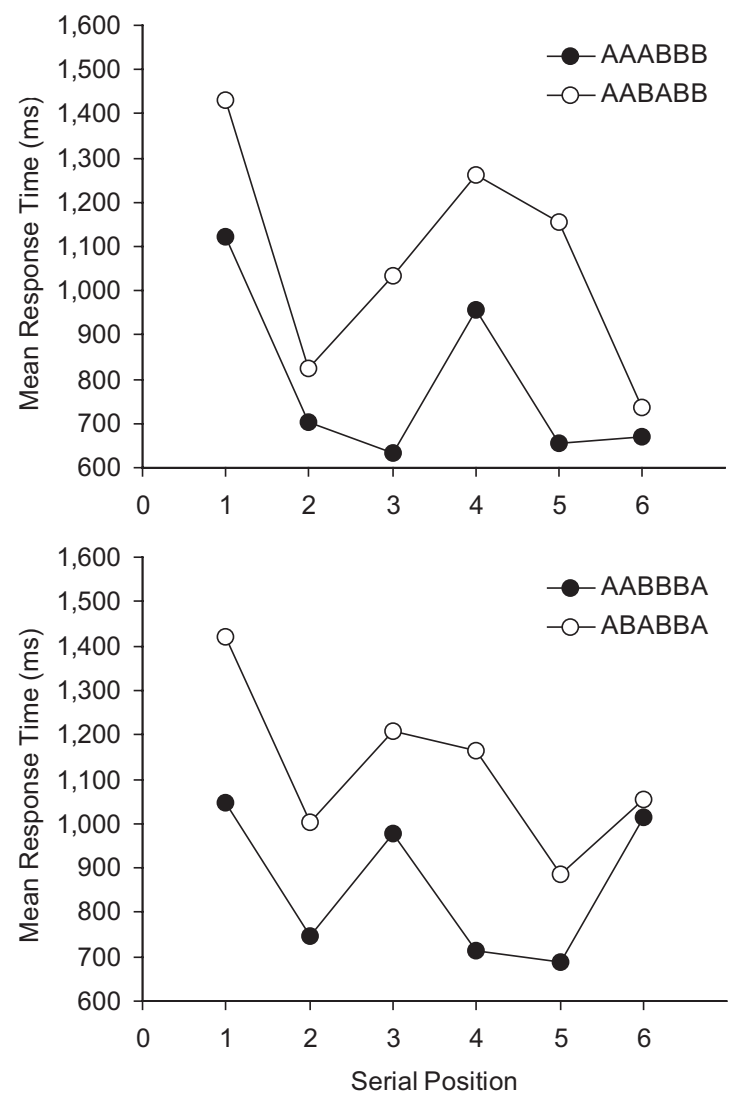

Figure 2. Mean response time for each serial position of each sequence in Experiment 2.

are not of particular interest; therefore, planned comparisons using the error term from the Sequence $\times$ Serial Position interaction are reported below.

Error rate analysis. Mean error rate was 3.3\% (see Table 3). Overall switch costs were $2.2 \%, 2.2 \%, 1.4 \%$, and $2.3 \%$ for the $\mathrm{AAABBB}, \mathrm{AABABB}, \mathrm{AABBBA}$, and $\mathrm{ABABBA}$ sequences, respectively, none of which was significantly different from any other, all $F \mathrm{~s}(1,285)<1.22, p \mathrm{~s}>.27$. Within-sequence switch costs were $2.1 \%, 2.5 \%, 1.2 \%$, and $2.1 \%$ for the AAABBB, AAB$\mathrm{ABB}, \mathrm{AABBBA}$, and $\mathrm{ABABBA}$ sequences, respectively, none of which was significantly different from any other, all $F_{\mathrm{s}}(1,285)<$ $2.35, p \mathrm{~s}>.12$. The lack of any differences among overall switch costs was likely due to the presence of a switch cost of $1.9 \%$ across sequences for the first serial position. These results would seem to indicate a lack of a relationship between the sequence and task levels with six-task sequences, but the RT data suggest otherwise.

RT analysis. Overall switch costs were $374,439,197$, and -46 ms for the AAABBB, AABABB, AABBBA, and ABABBA sequences, respectively, all of which were significantly different from each other, $F_{\mathrm{s}}(1,285)>20.40, p \mathrm{~s}<.001, \eta_{\mathrm{p}}^{2} \mathrm{~s}>.06$, except for the comparison of the $\mathrm{AAABBB}$ and $\mathrm{AABABB}$ sequences $(374 \mathrm{~ms}$ vs. $439 \mathrm{~ms}), F(1,285)=2.79, p=.10, \eta_{\mathrm{p}}^{2}=.01$. Consistent with the results of Experiment 1, the differences in overall switch costs were produced by sequence initiation time effects. RT profiles across serial position are plotted in Figure 2 for pairs of sequences involving the same task transition between iterations; RT was slowed at the first serial position relative to mean RT collapsed across subsequent serial positions for all sequences, $F \mathrm{~s}(1,285)>38.80, p \mathrm{~s}<.001, \eta_{\mathrm{p}}^{2} \mathrm{~s}>.11$.

There was no evidence in Experiment 2 that task repetitions were inherently associated with slower sequence initiation times than task switches. First serial position RT for the ABABBA sequence (a task repetition; $1,421 \mathrm{~ms}$ ) was a nonsignificant $10 \mathrm{~ms}$ faster than that of the AABABB sequence (a task switch; 1,431 $\mathrm{ms}), F(1,285)<1$. First serial position RT for the AABBBA sequence (a task repetition; 1,045 ms) was $76 \mathrm{~ms}$ faster than that of the AAABBB sequence (a task switch; 1,121 ms), a difference that was not statistically significant, $F(1,285)=2.79, p=.10$, $\eta_{\mathrm{p}}^{2}=.01$. Note that the two sequences in each comparison produce identical patterns of task transitions across an extended series of trials. The absence of statistically significant switch costs provides further evidence of hierarchical control from the sequence level to the task level, but these data do not support the reverse relationship: Task-level processing does not seem to affect sequence initiation.

Sequence complexity effects were examined by comparing first serial position RTs for pairs of sequences involving the same task transition between iterations but different numbers of withinsequence task switches. The $\mathrm{AAABBB}$ and $\mathrm{AABABB}$ sequences both involve a task switch between iterations, whereas the $\mathrm{AABBBA}$ and $\mathrm{ABABBA}$ sequences both involve a task repetition between iterations; these paired sequences are depicted in the top and bottom panels of Figure 2, respectively. First serial position $\mathrm{RT}$ for the AAABBB sequence (one within-sequence task switch) was $310 \mathrm{~ms}$ faster than that of the $\mathrm{AABABB}$ sequence (three within-sequence task switches), $F(1,285)=47.30, p<.001, \eta_{\mathrm{p}}^{2}=$ .14. First serial position RT for the AABBBA sequence (two within-sequence task switches) was $376 \mathrm{~ms}$ faster than that of the ABABBA sequence (four within-sequence task switches), $F(1$, 285) $=69.28, p<.001, \eta_{\mathrm{p}}^{2}=.20$.

Table 4

Analyses of Variance on Mean Response Time and Error Rate in Experiment 2

\begin{tabular}{|c|c|c|c|c|c|c|c|}
\hline \multirow[b]{2}{*}{ Effect } & \multirow[b]{2}{*}{$d f \mathrm{~s}$} & \multicolumn{3}{|c|}{ Response time } & \multicolumn{3}{|c|}{ Error rate } \\
\hline & & $F$ & $M S E$ & $\eta_{\mathrm{p}}^{2}$ & $F$ & $M S E$ & $\eta_{\mathrm{p}}^{2}$ \\
\hline Sequence $(\mathrm{S})$ & 3,57 & $80.93 * *$ & $38,434.19$ & .81 & 1.19 & 13.21 & .06 \\
\hline Serial position $(\mathrm{P})$ & 5,95 & $36.56 * *$ & $57,434.76$ & .66 & 0.66 & 8.16 & .03 \\
\hline $\mathrm{S} \times \mathrm{P}$ & 15,285 & $21.66^{* *}$ & $20,318.11$ & .53 & $4.19 * *$ & 8.52 & .18 \\
\hline
\end{tabular}

$* * p<.001$. 
These data indicate that sequence initiation times vary as a function of sequence complexity, suggesting that sequence initiation may involve preparation for the task transitions within the to-be-performed sequence, not just maintenance of a hierarchical control structure in working memory (De Jong, 1995; Lien \& Ruthruff, 2004). More within-sequence task switches require more preparation, resulting in slower sequence initiation times that reflect stronger effects of sequence-level processing. An important distinction between the present results and past research is that our sequence complexity effects do not reflect motor preparation (Henry \& Rogers, 1960; Rosenbaum et al., 1983) because the series of target stimuli-and hence the sequence of motor responses-was random across trials, both within and between iterations of a task sequence, so subjects could not have prepared motor responses in advance.

The differences in first serial position RTs between sequences of differing complexity are partly qualified by differences in overall $\mathrm{RT}$, which can be analyzed by comparing the within-sequence serial positions involving the same task transition for each paired sequence (i.e., Serial Positions 2, 4, and 6 involve the same task transitions in the AAABBB and $\mathrm{AABABB}$ sequences; Serial Positions 3, 5, and 6 involve the same task transitions in the AABBBA and ABABBA sequences; see Table 3). Mean RT for the relevant serial positions in the AAABBB sequence was $165 \mathrm{~ms}$ faster than that of the AABABB sequence, $F(1,285)=40.31, p<$ $.001, \eta_{\mathrm{p}}^{2}=.12$. Mean $\mathrm{RT}$ for the relevant serial positions in the AABBBA sequence was $157 \mathrm{~ms}$ faster than that of the ABABBA sequence, $F(1,285)=36.33, p<.001, \eta_{\mathrm{p}}^{2}=.11$.

There are two plausible interpretations of these effects. First, the effects may indicate that sequence complexity affects task-level performance beyond the first serial position. The overall shift in RT may not have been evident in Experiment 1 because the AABB and ABBA sequences were not sufficiently different to produce any shift. The variety of sequences used in Experiment 2 permitted a range of sequence complexity that may have enabled us to detect a shift. Second, the effects may reflect task transition frequency rather than sequence complexity. Recent studies have provided evidence that transition frequency affects task-switching performance (Monsell \& Mizon, 2006; Schneider \& Logan, 2006) and that a high frequency of task switches can elevate overall RT (Schneider \& Logan, 2006). Unfortunately, transition frequency and sequence complexity are confounded in our experiments; therefore, additional research with a modified experimental design is necessary to distinguish between these interpretations.

As in Experiment 1, it is useful to examine task-switching performance that is influenced to a lesser extent by sequence initiation. In Experiment 2, within-sequence switch costs were 292, 369, 280, and $221 \mathrm{~ms}$ for the AAABBB, AABABB, AABBBA, and ABABBA sequences, respectively, none of which was significantly different from any other, $F \mathrm{~s}(1,285)<2.78, p \mathrm{~s}>$ .09 , except for the comparison of the AABABB sequence with the AABBBA sequence (369 ms vs. $280 \mathrm{~ms}$ ), $F(1,285)=4.70, p<$ $.05, \eta_{\mathrm{p}}^{2}=.02$, and with the ABABBA sequence (369 ms vs. 221 $\mathrm{ms}), F(1,285)=10.30, p<.01, \eta_{\mathrm{p}}^{2}=.03$. The smaller differences in within-sequence switch costs suggest that beyond shifts in overall RT and sequence initiation times, the effects of hierarchical control on task-switching performance are attenuated within a sequence.

\section{Experiment 3}

Experiment 2 provided evidence that differences in sequence initiation time are related to sequence complexity, suggesting that sequence initiation may involve preparation for the task transitions in the to-be-performed sequence. Following up on this suggestion, the purpose of Experiment 3 was to examine how sequence initiation time is affected by preparation time.

Subjects performed the $\mathrm{AABB}$ and $\mathrm{ABBA}$ sequences from Experiment 1, except the response-stimulus interval (RSI) between successive trials was manipulated. The RSI preceding serial positions within the sequence (the within-sequence RSI) was shortened to $120 \mathrm{~ms}$, and the RSI preceding the first serial position of the sequence (the iteration $R S I$ ) was $500 \mathrm{~ms}$ or $1,000 \mathrm{~ms}$ in separate blocks. The RSI manipulation introduces temporal grouping of trials in accordance with the prescribed sequence, but it is clear from Experiment 1 that such grouping is not necessary to induce a hierarchical control structure in working memory (cf. Lien \& Ruthruff, 2004).

We expected that first serial position RT would be slower for the ABBA sequence than for the AABB sequence, replicating Experiment 1 . The main prediction concerned changes in first serial position RT with iteration RSI: If sequence initiation involves preparation for performance of a task sequence, then sequence initiation time should decrease as preparation time increases. Such an effect would be consistent with our view that the relationship between sequence- and task-level processing reflects hierarchical control.

\section{Method}

Subjects. Twenty students from Vanderbilt University completed the experiment in exchange for course credit or $\$ 5$. None of the subjects had taken part in Experiment 1 or 2.

Apparatus and stimuli. The apparatus and stimuli were identical to those in Experiments 1 and 2.

Procedure. The procedure was identical to that of Experiment 1 except for the manipulation of RSI: The within-sequence RSI was $120 \mathrm{~ms}$, and the iteration RSI was $500 \mathrm{~ms}$ or $1,000 \mathrm{~ms}$ in separate blocks. Consequently, the start of each iteration of the sequence was accompanied by a longer RSI; this point was mentioned to subjects in the instructions.

Subjects completed two practice blocks-one block for each sequence (AABB and $\mathrm{ABBA}$ ), with the task-specific sequence and iteration RSI randomly selected. Subjects then completed 16 experimental blocks-two blocks for each task-specific sequence with each iteration RSI, randomly selected with the restriction that each block type be used once every eight blocks. There were 40 trials per block, allowing 10 iterations of the relevant sequence and five presentations of each target. The trial format was identical to that of Experiments 1 and 2 except for the RSI manipulation. The experiment lasted about $25 \mathrm{~min}$.

\section{Results and Discussion}

Practice blocks and any blocks with error rates exceeding $20 \%$ ( $M=0.10$ blocks per subject) were excluded from all analyses. The first iteration of a sequence and trials with RTs below $100 \mathrm{~ms}$ or above $4,000 \mathrm{~ms}$ ( $M=1.16 \%$ of trials per subject) were also excluded from all analyses. Trials with errors were excluded from the RT analysis.

We collapsed across task-specific sequences for the AABB and ABBA sequences because they had identical patterns of data. Trials were classified as task switches or task repetitions, and then 
mean RT and error rate were calculated across subjects for each serial position in each sequence as a function of iteration RSI. These data are provided in Table 5, and mean RTs are plotted in Figure 3. The RT and error rate data were submitted to separate 2 (sequence) $\times 2$ (iteration $\mathrm{RSI}) \times 4$ (serial position) repeated measures ANOVAs, which are summarized in Table 6. The omnibus ANOVA effects are not of particular interest; therefore, planned comparisons using the relevant error terms from these ANOVAs are reported below.

Error rate analysis. Mean error rate was $1.9 \%$ (see Table 5). There was no main effect of iteration RSI, and it did not participate in any interactions (see Table 6); therefore, we collapsed across iteration RSI in our calculation of switch costs. Overall switch costs were $0.8 \%$ and $1.8 \%$ for the $\mathrm{AABB}$ and $\mathrm{ABBA}$ sequences, respectively, which were significantly different, $F(1,57)=4.30$, $p<.05, \eta_{\mathrm{p}}^{2}=.07$. As in Experiment 1 , the difference in overall switch costs was primarily due to the absence of a switch cost when comparing the first serial position of each sequence: The first serial position of the AABB sequence was a task switch with $1.4 \%$ error rate, and the first serial position of the ABBA sequence was a task repetition with $1.0 \%$ error rate, resulting in a nonsignificant switch cost of $0.4 \%, F(1,57)<1$. Within-sequence switch costs were $1.5 \%$ and $1.7 \%$ for the AABB and ABBA sequences, respectively, which were not significantly different, $F(1,57)<1$. These data replicate those of Experiment 1 and demonstrate the effects of hierarchical control on error rate at sequence transitions. The lack of a main effect or any interactions involving iteration RSI indicates that preparation time did not affect error rate, but the next analysis shows that it had a strong effect on RT.

$R T$ analysis. Overall switch costs and within-sequence switch costs for each sequence did not differ between iteration RSIs, all $F_{\mathrm{s}}(1,57)<1.75, p \mathrm{~s}>.18$. Overall switch costs (collapsed across iteration RSI) were $446 \mathrm{~ms}$ and $-11 \mathrm{~ms}$ for the AABB and ABBA sequences, respectively, which were significantly different, $F(1$, $57)=609.87, p<.001, \eta_{\mathrm{p}}^{2}=.91$. First serial position RT for each sequence was slower than the mean RT collapsed across subsequent serial positions, $F \mathrm{~s}(1,57)>673.81, p \mathrm{~s}<.001, \eta_{\mathrm{p}}^{2} \mathrm{~s}>.91$. The difference in overall switch costs was primarily due to differ-

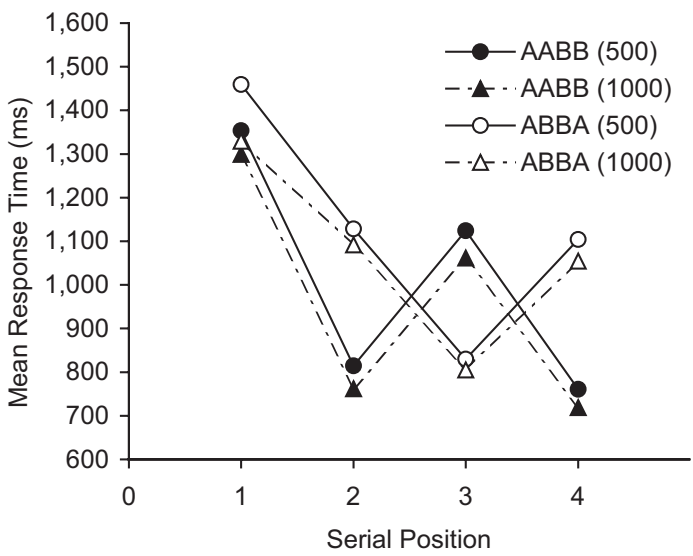

Figure 3. Mean response time for each serial position of each sequence as a function of iteration response-stimulus interval (in milliseconds; in parentheses) in Experiment 3.

ences in sequence initiation time (see Figure 3): First serial position RT for the ABBA sequence was $68 \mathrm{~ms}$ slower than that of the AABB sequence, a significant difference, $F(1,57)=13.55, p<$ $.001, \eta_{\mathrm{p}}^{2}=.19$. These data replicate the pattern of overall switch costs and sequence initiation times observed in Experiment 1. Within-sequence switch costs (collapsed across iteration RSI) were $329 \mathrm{~ms}$ and $277 \mathrm{~ms}$ for the $\mathrm{AABB}$ and $\mathrm{ABBA}$ sequences, respectively, which were significantly different, $F(1,57)=5.18$, $p<.05, \eta_{\mathrm{p}}^{2}=.08$. The significant difference in within-sequence switch costs is in contrast to the nonsignificant difference observed in Experiment 1 for reasons that are unclear.

The critical data in Experiment 3 concern the effect of iteration RSI on first serial position RT. For the AABB sequence, first serial position RT decreased $54 \mathrm{~ms}$ as the iteration RSI increased from 500 to $1,000 \mathrm{~ms}, F(1,57)=4.30, p<.05, \eta_{\mathrm{p}}^{2}=.07$. For the ABBA sequence, first serial position RT decreased $130 \mathrm{~ms}$ as the iteration RSI increased from 500 to $1,000 \mathrm{~ms}, F(1,57)=24.68$, $p<.001, \eta_{\mathrm{p}}^{2}=.30$. The difference between sequences in the

Table 5

Task Transition, Mean Response Time (RT; in Milliseconds), and Error Rate in Experiment 3

\begin{tabular}{|c|c|c|c|c|c|}
\hline \multirow{2}{*}{$\begin{array}{c}\text { Sequence and } \\
\text { iteration RSI }\end{array}$} & \multirow[b]{2}{*}{ Measure } & \multicolumn{4}{|c|}{ Serial position } \\
\hline & & 1 & 2 & 3 & 4 \\
\hline \multicolumn{6}{|l|}{$\mathrm{AABB}$} \\
\hline \multirow{3}{*}{500} & Task transition & TS & TR & TS & TR \\
\hline & RT & $1,353(61)$ & $814(45)$ & $1,124(37)$ & $761(35)$ \\
\hline & Error rate & $1.4(0.3)$ & $0.7(0.3)$ & $3.2(0.7)$ & $1.5(0.6)$ \\
\hline \multirow[t]{3}{*}{1,000} & Task transition & $\mathrm{TS}$ & TR & TS & TR \\
\hline & RT & $1,299(56)$ & $762(43)$ & $1,061(43)$ & $719(38)$ \\
\hline & Error rate & $1.5(0.5)$ & $1.0(0.3)$ & $2.7(0.6)$ & $2.5(0.6)$ \\
\hline \multicolumn{6}{|l|}{ ABBA } \\
\hline \multirow[t]{3}{*}{500} & Task transition & TR & TS & TR & TS \\
\hline & RT & $1,459(74)$ & $1,129(33)$ & $830(48)$ & $1,104(36)$ \\
\hline & Error rate & $0.9(0.4)$ & $2.7(0.7)$ & $1.3(0.6)$ & $2.7(0.8)$ \\
\hline \multirow{3}{*}{1,000} & Task transition & TR & TS & TR & TS \\
\hline & RT & $1,329(66)$ & $1,092(35)$ & $805(44)$ & $1,055(41)$ \\
\hline & Error rate & $1.1(0.4)$ & $3.5(0.9)$ & $1.1(0.5)$ & $2.5(0.5)$ \\
\hline
\end{tabular}

Note. $\quad$ Standard errors appear in parentheses. RSI $=$ response-stimulus interval; $\mathrm{TS}=$ task switch; $\mathrm{TR}=$ task repetition. 
Table 6

Analyses of Variance on Mean Response Time and Error Rate in Experiment 3

\begin{tabular}{|c|c|c|c|c|c|c|c|}
\hline \multirow[b]{2}{*}{ Effect } & \multirow[b]{2}{*}{$d f \mathrm{~s}$} & \multicolumn{3}{|c|}{ Response time } & \multicolumn{3}{|c|}{ Error rate } \\
\hline & & $F$ & $M S E$ & $\eta_{\mathrm{p}}^{2}$ & $F$ & $M S E$ & $\eta_{\mathrm{p}}^{2}$ \\
\hline Sequence $(\mathrm{S})$ & 1,19 & $86.74 * *$ & $11,914.57$ & .82 & 0.28 & 6.39 & .01 \\
\hline Iteration RSI (I) & 1,19 & $25.46 * *$ & $10,058.61$ & .57 & 0.57 & 5.43 & .03 \\
\hline Serial position $(\mathrm{P})$ & 3,57 & $95.84 * *$ & $37,575.36$ & .84 & $4.75^{*}$ & 3.81 & .20 \\
\hline $\mathrm{S} \times \mathrm{I}$ & 1,19 & 0.16 & $7,252.74$ & .01 & 0.00 & 3.32 & .00 \\
\hline $\mathrm{S} \times \mathrm{P}$ & 3,57 & $74.28 * *$ & $22,258.82$ & .80 & $9.73 * *$ & 5.94 & .34 \\
\hline $\mathrm{I} \times \mathrm{P}$ & 3,57 & 1.49 & $7,544.64$ & .07 & 0.36 & 8.57 & .02 \\
\hline $\mathrm{S} \times \mathrm{I} \times \mathrm{P}$ & 3,57 & 1.76 & $6,841.77$ & .09 & 0.71 & 4.56 & .04 \\
\hline
\end{tabular}

Note. $\quad$ RSI $=$ response-stimulus interval.

$* p<.05 . \quad * * p<.001$.

reduction of first serial position RT with iteration RSI was significant, $F(1,57)=4.19, p<.05, \eta_{\mathrm{p}}^{2}=.07$, reflecting the fact that the significant difference between first serial position RTs at the 500-ms iteration RSI (a 106-ms "switch benefit") became a nonsignificant difference at the 1,000-ms iteration RSI (see Figure 3), $F(1,57)=1.34, p=.25$. Note that there was no switch cost at the first serial position for either iteration RSI, providing further evidence of hierarchical control that is consistent with Experiments 1 and 2 .

These data indicate that sequence initiation time can be reduced by increasing preparation time: Some of the time associated with sequence initiation was absorbed in the longer iteration RSI, leading to faster first serial position RTs, even in the absence of external cues (cf. Luria \& Meiran, 2003). Doubling the iteration RSI did not eliminate the sequence initiation time for either sequence, but it is possible that a longer iteration RSI could be more effective. These results support the idea that sequence initiation involves preparation for performance of the task sequence and can be interpreted as additional evidence of hierarchical control.

An unexpected finding in Experiment 3 was that the iteration RSI affected performance beyond the first serial position. Collapsing across sequences and excluding the first serial position, mean RT was $45 \mathrm{~ms}$ faster with the 1,000-ms iteration RSI compared with the 500-ms iteration RSI (see Figure 3), even though the within-sequence RSI was always $120 \mathrm{~ms}$. The main effect of iteration RSI from the omnibus ANOVA results reported in Table 6 remained significant in a separate 2 (sequence) $\times 2$ (iteration RSI) $\times 3$ (serial position, excluding the first serial position) repeated measures ANOVA, $F(1,19)=15.04, M S E=7,989.84$, $p<.01, \eta_{\mathrm{p}}^{2}=.44$. This carryover effect of iteration RSI indicates that the preparation involved in sequence initiation can modulate performance of the entire sequence, suggesting that the degree to which a hierarchical control structure is instantiated and maintained in working memory is related to the amount of time available for preparing the hierarchical control structure. ${ }^{5}$

\section{Experiment 4}

As noted earlier, we expected the effects of hierarchical control to be manifest at sequence transitions. In Experiments 1-3, subjects performed the same sequence repeatedly and we focused on the first serial position of sequence repetitions. The purpose of Experiment 4 was to extend our findings to sequence switches, to determine whether sequence transitions mirror task transitions: Is there a cost associated with switching sequences?

Luria and Meiran (2003) and Lien and Ruthruff (2004) found sequence switch costs in dual-task and task-switching performance, respectively, but their experiments typically involved only two-task sequences and subjects could rely on external cues rather than sequence information in memory to switch sequences. By modifying the design of Experiments 1-3, we were able to analyze sequence switches and sequence repetitions for longer sequences in the absence of external cues in Experiment 4, allowing us to attribute any effects to the implementation and maintenance of a hierarchical control structure in working memory.

Subjects memorized task-specific AABB and ABBA sequences and then performed the sequences according to a memorized supersequence (a sequence of sequences) that differed between blocks. Within each supersequence there were sequence repetitions and sequence switches, allowing us to examine sequence initiation times for both types of sequence transitions. If switching sequences requires more preparation (i.e., more extensive sequencelevel processing) than repeating a sequence, then first serial position RTs should be greater for sequence switches than for sequence repetitions, with these sequence switch costs exaggerating differences in overall switch cost. Moreover, there should be no switch cost at the first serial position when switching sequences, because changing the hierarchical control structure in working memory should strongly affect task-level processing. The presence of sequence switch costs, perturbed overall switch costs, and no switch cost at the first serial position when repeating and switching sequences would provide further evidence in support of the idea

\footnotetext{
${ }^{5}$ An issue in this experiment is whether the reduction in first serial position RT with increased iteration RSI exceeded the reduction in RT for subsequent serial positions. For the ABBA sequence, the reduction in first serial position RT of $130 \mathrm{~ms}$ was significantly greater than the reduction in RT for subsequent serial positions of $37 \mathrm{~ms}, F(1,57)=9.44, p<.01, \eta_{\mathrm{p}}^{2}=$ .14. For the AABB sequence, the reduction in first serial position RT of 54 ms was not significantly greater than the reduction in RT for subsequent serial positions of $52 \mathrm{~ms}, F(1,57)<1$. We speculate that the lack of a differential reduction in RT across serial positions for the AABB sequence may reflect a floor effect. Sequence initiation time may have already been at a near-asymptotic level for the AABB sequence; therefore, an increased iteration RSI would not have yielded a differential reduction in RT.
} 
that task-switching performance is under hierarchical control in our experiments.

\section{Method}

Subjects. Twenty-four students from Vanderbilt University completed the experiment in exchange for course credit or $\$ 10$. None of the subjects had taken part in Experiments 1-3.

Apparatus and stimuli. The apparatus and stimuli were identical to those of Experiments 1-3.

Procedure. The procedure from Experiment 1 was modified to enable sequence repetitions and sequence switches within the same block of trials. Subjects were instructed to memorize two sequences. The sequences were labeled $A$ and $B$ in the experiment, but we refer to them as $X$ and $Y$ to avoid confusing supersequences with their constituent sequences. For half of the subjects, Sequence X was CCSS and Sequence $\mathrm{Y}$ was CSSC; for the other half, Sequence X was SSCC and Sequence Y was SCCS. Note that the CCSS and SSCC sequences are task-specific AABB sequences, and the CSSC and SCCS sequences are task-specific ABBA sequences. Subjects completed six practice blocks of trials to facilitate memorization of the sequences. Each practice block began with a screen that reminded subjects about the $\mathrm{X}$ and $\mathrm{Y}$ sequences. The next screen displayed a string of four letters that indicated how many times one of the sequences was to be performed during the practice block (e.g., $X X X X$ indicated that Sequence $\mathrm{X}$ was to be performed four times). Subjects then initiated the block. There were 16 trials per block, allowing two presentations of each target. The trial format was identical to that of Experiments 1 and 2 except the RSI for all trials was $120 \mathrm{~ms}$.

Following the practice blocks, subjects received instructions concerning the experimental blocks. The experimental blocks were identical in format to the practice blocks, except the string of four letters at the start of each block represented one of four supersequences (i.e., a sequence of the $X$ and Y sequences): XXYY, YYXX, XYYX, and YXXY. Note that within each supersequence, there were sequence repetitions (e.g., $\mathrm{XX}$ ) and sequence switches (e.g., XY). Sequence repetitions and sequence switches occurred with equal frequency across supersequences. Subjects were instructed to perform the $\mathrm{X}$ and $\mathrm{Y}$ sequences in the order indicated by the supersequence (e.g., XYYX indicated that Sequence $\mathrm{X}$ was to be performed first, then Sequence $\mathrm{Y}$ twice, then Sequence $\mathrm{X}$ to finish the block). The experimenter stressed the importance of remembering the $\mathrm{X}$ and $\mathrm{Y}$ sequences and the relevant supersequence during each block. The trial format was unchanged from the practice blocks. Subjects completed 2 practice blocks under these conditions- one block involving the XXYY or YYXX supersequence and one block involving the XYYX or YXXY supersequence. Subjects then completed 48 experimental blocks-12 blocks for each supersequence, randomly selected with no restrictions. The experiment lasted about 45 $\min$.

\section{Results and Discussion}

Practice blocks and any blocks with error rates exceeding $20 \%$ ( $M=1.17$ blocks per subject) were excluded from all analyses. The first sequence in each supersequence and trials with RTs below $100 \mathrm{~ms}$ or above $4,000 \mathrm{~ms}$ ( $M=3.64 \%$ of trials per subject $)$ were also excluded from all analyses. Trials with errors were excluded from the RT analysis.

We collapsed across supersequences and classified task-specific sequences as AABB or ABBA sequences. Sequences were classified as sequence switches or sequence repetitions according to the relationship between the current and immediately preceding sequences in the supersequence. Trials were classified as task switches or task repetitions, and then mean RT and error rate were calculated across subjects for each serial position in each sequence as a function of sequence transition. These data are provided in Table 7, and mean RTs are plotted in Figure 4. The RT and error rate data were submitted to separate 2 (sequence) $\times 2$ (sequence transition) $\times 4$ (serial position) repeated measures ANOVAs, which are summarized in Table 8 . The omnibus ANOVA effects are not of particular interest; therefore, planned comparisons based on the relevant error terms from these ANOVAs are reported below.

Error rate analysis. Mean error rate was $1.7 \%$ (see Table 7). For sequence repetitions, overall switch costs were $0.0 \%$ and $1.2 \%$ for the $\mathrm{AABB}$ and $\mathrm{ABBA}$ sequences, respectively, which were significantly different, $F(1,69)=5.50, p<.05, \eta_{\mathrm{p}}^{2}=.07$. Replicating Experiments 1 and 3, there was a nonsignificant switch cost of $0.1 \%$ between first serial positions, $F(1,69)<1$. Withinsequence switch costs were $0.4 \%$ and $1.1 \%$ for the $\mathrm{AABB}$ and ABBA sequences, respectively, which were not significantly different, $F(1,69)=1.16, p=.29$. For sequence switches, overall switch costs were $-1.8 \%$ and $2.7 \%$ for the $\mathrm{AABB}$ and $\mathrm{ABBA}$

Table 7

Task Transition, Mean Response Time (RT; in Milliseconds), and Error Rate in Experiment 4

\begin{tabular}{|c|c|c|c|c|c|}
\hline \multirow{2}{*}{$\begin{array}{c}\text { Sequence and } \\
\text { sequence transition }\end{array}$} & \multirow[b]{2}{*}{ Measure } & \multicolumn{4}{|c|}{ Serial position } \\
\hline & & 1 & 2 & 3 & 4 \\
\hline \multicolumn{6}{|l|}{ AABB } \\
\hline \multirow[t]{3}{*}{ Sequence switch } & Task transition & TS & TR & TS & TR \\
\hline & RT & $2,127(85)$ & $885(50)$ & 969 (48) & $832(42)$ \\
\hline & Error rate & $1.4(0.5)$ & $2.9(0.7)$ & $1.1(0.4)$ & $3.2(0.8)$ \\
\hline \multirow[t]{3}{*}{ Sequence repetition } & Task transition & TS & TR & TS & TR \\
\hline & RT & $1,496(62)$ & $889(34)$ & $1,063(44)$ & $885(40)$ \\
\hline & Error rate & $0.9(0.4)$ & $1.1(0.3)$ & $1.8(0.5)$ & $1.7(0.4)$ \\
\hline \multicolumn{6}{|l|}{ ABBA } \\
\hline \multirow[t]{3}{*}{ Sequence switch } & Task transition & TR & TS & TR & TS \\
\hline & $\mathrm{RT}$ & $2,112(84)$ & $1,169(47)$ & $878(40)$ & $1,072(41)$ \\
\hline & Error rate & $0.9(0.3)$ & $2.9(0.7)$ & $0.5(0.3)$ & $3.8(0.8)$ \\
\hline \multirow[t]{3}{*}{ Sequence repetition } & Task transition & TR & TS & TR & TS \\
\hline & RT & $1,665(65)$ & $1,150(45)$ & $876(42)$ & $1,139(50)$ \\
\hline & Error rate & $0.8(0.3)$ & $2.4(0.7)$ & $1.0(0.4)$ & $1.8(0.5)$ \\
\hline
\end{tabular}

Note. Standard errors appear in parentheses. TS $=$ task switch; TR $=$ task repetition. 


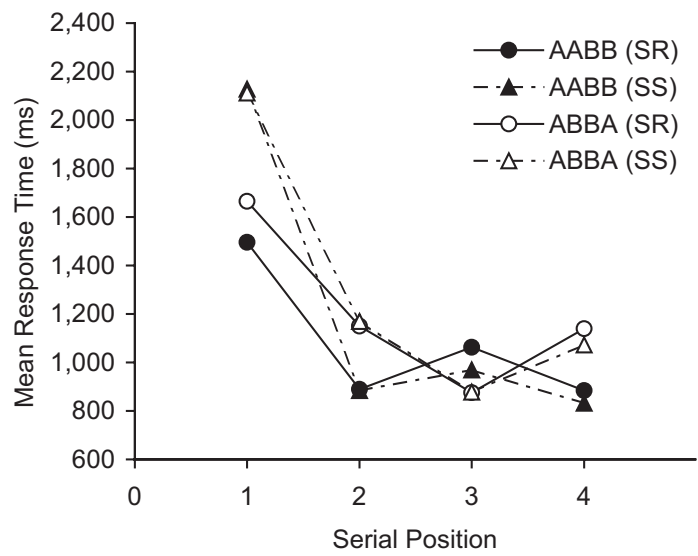

Figure 4. Mean response time for each serial position of each sequence as a function of sequence transition (in parentheses; $\mathrm{SR}=$ sequence repetition, $\mathrm{SS}=$ sequence switch) in Experiment 4.

sequences, respectively, which were significantly different, $F(1$, $69)=69.45, p<.001, \eta_{\mathrm{p}}^{2}=.50$. Consistent with the sequence repetition data, there was a nonsignificant switch cost of $0.5 \%$ between first serial positions, $F(1,69)<1$. Within-sequence switch costs were $-1.9 \%$ and $2.9 \%$ for the AABB and ABBA sequences, respectively, which were significantly different, $F(1$, $69)=54.13, p<.001, \eta_{\mathrm{p}}^{2}=.44$, suggesting that sequence switches had effects on error rate beyond the first serial position. Regarding potential sequence switch costs in error rate, the difference between first serial positions for sequence switches and sequence repetitions was $0.5 \%$ for the $\mathrm{AABB}$ sequence and $0.1 \%$ for the ABBA sequence, both nonsignificant differences, $F \mathrm{~s}(1$, $69)<1$. The error rate data are consistent with previous results and provide evidence of hierarchical control.

$R T$ analysis. For sequence repetitions, overall switch costs were $393 \mathrm{~ms}$ and $-125 \mathrm{~ms}$ for the $\mathrm{AABB}$ and $\mathrm{ABBA}$ sequences, respectively, which were significantly different, $F(1,69)=$ $317.44, p<.001, \eta_{\mathrm{p}}^{2}=.82$. First serial position RT for each sequence was slower than the mean RT collapsed across subsequent serial positions, $F \mathrm{~s}(1,69)>537.19, p \mathrm{~s}<.001, \eta_{\mathrm{p}}^{2} \mathrm{~s}>.88$. These effects replicate the corresponding results from Experiments 1 and 3. For sequence switches, overall switch costs were $690 \mathrm{~ms}$ and $-374 \mathrm{~ms}$ for the $\mathrm{AABB}$ and $\mathrm{ABBA}$ sequences, respectively, which were significantly different, $F(1,69)=1,336.80, p<.001$, $\eta_{\mathrm{p}}^{2}=.95$. The difference in overall switch costs was much larger for sequence switches than for sequence repetitions, suggesting that hierarchical control was more pronounced for sequence switches.

The substantial differences in overall switch costs were due to sequence switch costs: First serial position RTs were slower for sequence switches compared with sequence repetitions (see Table 7 and Figure 4). For the AABB sequence, first serial position RT was $631 \mathrm{~ms}$ slower for sequence switches compared with sequence repetitions, $F(1,69)=470.62, p<.001, \eta_{\mathrm{p}}^{2}=.87$. For the ABBA sequence, first serial position RT was $447 \mathrm{~ms}$ slower for sequence switches compared with sequence repetitions, $F(1,69)=236.00$, $p<.001, \eta_{\mathrm{p}}^{2}=.77$. The difference between the AABB and ABBA sequences in the magnitude of these sequence switch costs was significant, $F(1,69)=20.04, p<.001, \eta_{\mathrm{p}}^{2}=.23$. These data indicate that sequence initiation time depends on the sequence transition: More sequence-level processing is required for sequence switches than for sequence repetitions, resulting in sequence switch costs.

The sequence switch costs can be explained by considering what must be involved with sequence initiation for both sequence transitions. For sequence repetitions, the hierarchical control structure in working memory can be retained for performance of the next sequence, facilitating sequence initiation. For sequence switches, the hierarchical control structure in working memory has to be changed to accommodate a different sequence. Switching hierarchical control structures is arguably more time consuming than maintaining an existing hierarchical control structure; therefore, sequence initiation would take longer for sequence switches than for sequence repetitions.

Differences in sequence initiation time between the $\mathrm{AABB}$ and ABBA sequences can also be examined for each sequence transition. For sequence repetitions, first serial position RT for the ABBA sequence was $168 \mathrm{~ms}$ slower than that of the AABB sequence, a significant difference, $F(1,69)=33.45, p<.001$, $\eta_{\mathrm{p}}^{2}=.33$, replicating Experiments 1 and 3. For sequence switches, first serial position $\mathrm{RT}$ for the $\mathrm{ABBA}$ sequence was a nonsignificant $16 \mathrm{~ms}$ faster than that of the AABB sequence, $F(1,69)<1$. An absence of switch cost at the task level for either sequence repetitions or sequence switches represents additional evidence of hierarchical control-maintaining or changing the hierarchical control structure altered the contents of working memory in a way that eliminated switch cost.

The effects of sequence transition on task-switching performance were attenuated beyond the first serial position (see Figure

Table 8

Analyses of Variance on Mean Response Time and Error Rate in Experiment 4

\begin{tabular}{|c|c|c|c|c|c|c|c|}
\hline \multirow[b]{2}{*}{ Effect } & \multirow[b]{2}{*}{$d f_{s}$} & \multicolumn{3}{|c|}{ Response time } & \multicolumn{3}{|c|}{ Error rate } \\
\hline & & $F$ & $M S E$ & $\eta_{\mathrm{p}}^{2}$ & $F$ & $M S E$ & $\eta_{\mathrm{p}}^{2}$ \\
\hline Sequence $(\mathrm{S})$ & 1,23 & $58.03 * *$ & $21,571.27$ & .72 & 0.00 & 9.56 & .00 \\
\hline Sequence transition $(\mathrm{T})$ & 1,23 & $65.33^{* * *}$ & $17,833.92$ & .74 & 3.36 & 12.67 & .13 \\
\hline Serial position $(\mathrm{P})$ & 3,69 & $155.88 * *$ & $116,095.20$ & .87 & $9.80 * *$ & 6.86 & .30 \\
\hline $\mathrm{S} \times \mathrm{T}$ & 1,23 & 0.60 & $15,645.85$ & .03 & 0.19 & 5.92 & .01 \\
\hline $\mathrm{S} \times \mathrm{P}$ & 3,69 & $27.47 * *$ & $31,700.81$ & .54 & 2.01 & 4.89 & .08 \\
\hline $\mathrm{T} \times \mathrm{P}$ & 3,69 & 89.38 ** & $22,183.77$ & .80 & $4.09 *$ & 6.24 & .15 \\
\hline $\mathrm{S} \times \mathrm{T} \times \mathrm{P}$ & 3,69 & $8.35 * *$ & $10,158.89$ & .27 & 1.05 & 3.44 & .04 \\
\hline
\end{tabular}

$* p<.05 . \quad * * p<.001$ 
4). In a 2 (sequence) $\times 2$ (sequence transition) $\times 3$ (serial position, excluding the first serial position) repeated measures ANOVA, the main effect of sequence transition, the Sequence Transition $\times$ Serial Position interaction, and the Sequence $\times$ Sequence Transition $\times$ Serial Position interaction-which were all highly significant in the original ANOVA (see Table 8) - only approached significance, all $F \mathrm{~s}<3.81, p \mathrm{~s}>.06$. These marginally significant effects are reflected in the within-sequence switch costs. For the AABB sequence, within-sequence switch costs were $111 \mathrm{~ms}$ and $176 \mathrm{~ms}$ for sequence switches and sequence repetitions, respectively, which were almost significantly different, $F(1,69)=3.38$, $p=.07, \eta_{\mathrm{p}}^{2}=.05$. For the ABBA sequence, within-sequence switch costs were $243 \mathrm{~ms}$ and $269 \mathrm{~ms}$ for sequence switches and sequence repetitions, respectively, which were not significantly different, $F(1,69)<1$. These results are consistent with some of the data from Lien and Ruthruff (2004) and indicate that sequence transition effects are associated primarily with sequence initiation.

\section{General Discussion}

Hierarchical control of cognitive processes in task-switching performance was established by investigating the relationship between sequence- and task-level processing when subjects performed different tasks that were organized in explicit, memorized sequences. Hierarchical control was inferred from two main findings: (a) sequence initiation time effects-slower RTs for the first serial position of a sequence relative to subsequent serial positions-that were related to sequence complexity, preparation time, and sequence transition; and (b) an absence of switch cost at the first serial position across sequences. The sequence-level processing associated with repeating or switching sequences seems to eliminate task-level effects at the first serial position, suggesting that task performance is strongly linked to the maintenance or instantiation of a hierarchical control structure in working memory. In the remainder of this article, we discuss how these findings relate to research on motor programs, serial memory, task set, and control.

\section{Motor Programs, Serial Memory, and the Domain Generality of Hierarchical Control}

Motor programs. Since the time of Lashley (1951) and Miller et al. (1960), many researchers have recognized the importance of plans in producing complex patterns of behavior. Plans and hierarchical control have been studied extensively in the motor programming literature (for reviews, see Keele et al., 1990; Keele \& Summers, 1976; Rosenbaum, 1984, 1987), and there are many parallels to the present study, including sequence initiation time effects and preparation effects related to sequence complexity.

Studies of simple motor programming (e.g., performing fingertapping sequences) have been conducted by many authors (e.g., Povel \& Collard, 1982; Restle \& Burnside, 1972; Rosenbaum et al., 1983; see also Koch \& Hoffmann, 2000) and have generated three findings related to hierarchical control that are mirrored in the present study: (a) Different sequences yield different RT profiles, even if they result in identical patterns of motor responses across an extended series of trials; (b) peaks in the RT profiles occur at positions corresponding to chunks of a hierarchical structure; and (c) response repetition effects coinciding with sequence or chunk transitions are sometimes perturbed. In our experiments, we also found that different sequences yielded different RT profiles, with peaks at the first serial position of each sequence and altered task transition effects at sequence transitions.

Studies of more complex motor programming (e.g., typing and speaking) have provided additional evidence of hierarchical control (e.g., Gordon \& Meyer, 1987) and preparation effects related to sequence (or stimulus) complexity (e.g., Klapp et al., 1973; Sternberg et al., 1978). Henry and Rogers (1960) showed that simple RT for initiating a motor action increased as the complexity of the action increased. Klapp et al. (1973) demonstrated that the time to begin speaking a word was longer if the word had two syllables compared with one syllable, even if subjects were naming pictures (which are not inherently associated with syllables). Related findings have been reported by Sternberg et al. (1978) for lists of words and Rosenbaum (1987) for finger-tapping sequences. Preparation effects based on complexity suggest planning of an entire utterance or sequence that may involve access to each element or chunk (e.g., syllable or task name) in a hierarchical representation, an idea that resonates with Henry and Rogers's "memory drum" theory of action. They suggested that the information for performing a motor action is stored as a program in a form of motor memory and that the time to initiate a movement reflected the time required to transmit a motor program to effectors, with more complicated movements involving more comprehensive programs.

Serial memory. The relationship between plans and memory is not restricted to motor programming; many researchers have also recognized the importance of plans in memory retrieval. Hierarchical organization of items in memory and its effects on recall have been studied extensively in the memory literature (for reviews, see Bower, 1970; Johnson, 1970), but much of the evidence is restricted to accuracy measures. However, parallels to the present study in terms of sequence and chunk initiation time effects have surfaced recently in the domain of serial memory.

A few studies have focused on RT (i.e., interresponse time [IRT]) in addition to accuracy in the serial recall of digits or letters. In an experiment conducted to establish a set of basic parameters for a model of serial recall (discussed below), Anderson and Matessa (1997) had subjects recall digit sequences that were spatially grouped into chunks during presentation. They observed slower IRTs at the start of each sequence and chunk in forward recall, and these initiation time effects increased as sequence length increased. Anderson et al. (1998) extended these earlier findings with a similar but expanded experiment involving a greater variety of sequence lengths and both forward and backward recall. They found robust sequence and chunk initiation time effects for recall in either direction. Kahana and Jacobs (2000) explored IRTs for serial recall of long lists of consonants that had been memorized to perfection in the absence of instructions to chunk items. Despite variability in the chunking strategies spontaneously adopted by subjects, Kahana and Jacobs obtained clear evidence of sequence and chunk initiation time effects in both forward and backward recall. Collectively, these findings represent evidence of hierarchical control, because the timing of recall closely followed the structure of the sequence in memory, consistent with the sequence initiation time effects in the motor programming literature and in the present study.

Domain generality. Although there are parallels between the present study and research on motor programming and serial memory, a critical distinction is that the present study dealt with 
the performance of task sequences rather than the performance of motor sequences or the simple recall of stimulus sequences. The sequences of motor responses in our experiments were unpredictable because the sequences of target stimuli varied randomly across sequence transitions. Consequently, the sequence initiation time effects in our experiments cannot be attributed to the sequencing of motor actions or target stimuli. Instead, our sequence initiation time effects - and especially the recurrent absence of a switch cost at the first serial position-must be attributed to the sequencing of tasks at a higher and more abstract level of processing.

The distinction between task sequences and motor sequences in particular raises an important caveat: Hierarchical control in the cognitive system does not necessarily have to map onto hierarchical control in the motor system. However, the fact that many similarities exist between the cognitive and motor levels suggests that the hierarchical organization of control tends to be general and not domain specific (Rosenbaum, 1987; see also Cohen, 2000), ranging from task switching to serial memory to motor control. This domain generality gives way to the idea that the performance of different tasks can be fractionated into an intricate system of hierarchies and subhierarchies, from the abstract task level to the fine-grained motor level (Miller et al., 1960). The hierarchical control structure for a task sequence is likely composed of many subhierarchies, not only for each task but also for specific motor responses.

The idea of nested hierarchies was important to Miller et al. (1960), who suggested that plans are built from hierarchies of basic functional units called test-operate-test-exit (TOTE) units. Each operation within a TOTE unit can be represented by a hierarchy of other TOTE units, enabling the construction of plans for complex patterns of behavior. Nested hierarchies remain prominent in current theorizing about the control of behavior, as evident in recent computational models of routine action control (Cooper \& Shallice, 2000, in press; but see Botvinick \& Plaut, 2004). For example, a hierarchically organized network of goal-directed action schemas forms the core of Cooper and Shallice's (2000) formal implementation of the contention-scheduling system proposed by Norman and Shallice (1986), suggesting that nested hierarchies are useful constructs for understanding different levels of control.

\section{Task Set}

Although the concept of set has existed for decades in psychology, its meaning is unclear (for early reviews, see Dashiell, 1940; Gibson, 1941), and definitions of task set and task-set reconfiguration in contemporary psychology tend to be infrequent and vague. Rogers and Monsell (1995) wrote that "to adopt a task-set is to select, link, and configure the elements of a chain of processes that will accomplish a task" (p. 208), and Mayr and Keele (2000) proposed that task sets "specify the configuration of perceptual, attentional, mnemonic, and motor processes critical for a particular task goal" (p. 5). Adopting a different task set involves task-set reconfiguration-“a sort of mental 'gear changing"' (Monsell, 2003 , p. 135) that engages some combination of executive control processes. These definitions have evoked some criticism (Altmann, 2003; Schneider \& Logan, 2005), but a general point to be taken from them is that the focus is on individual tasks-even though what constitutes a "task" is debatable.
The modern concept of task set can be refined by thinking of a task set as a plan (Miller et al., 1960) and assuming hierarchical organization not just of tasks (e.g., Kleinsorge \& Heuer, 1999; Kleinsorge et al., 2004) but also of task sequences (e.g., De Jong, 1995; Lien \& Ruthruff, 2004; see also Luria \& Meiran, 2003). We argue that the hierarchical control structure for a task sequence constitutes part of the task set-a plan for controlling behaviorand that the sequence initiation times and related effects observed in our experiments reflect preparation associated with instantiating and maintaining the hierarchical control structure in working memory. Changing the hierarchical control structure can be interpreted as a form of task-set reconfiguration, but at the level of task sequences instead of individual tasks.

The idea that the hierarchical control structure for a task sequence constitutes part of the task set is reminiscent of research by Luchins and Luchins (1950) on problem solving. In their experiments, subjects had to determine how to obtain a specific volume of fluid by pouring fluid into and out of containers that varied in size. The first few problems could all be solved by the same sequence of operations, which was referred to as the Einstellung method-“a special kind of mental set" (Luchins \& Luchins, 1950, p. 279). In contrast to the modern emphasis on flexibility in changing task set, Luchins and Luchins focused on the rigidity of task set by noting that many subjects were unable to abandon the Einstellung method for later problems that could be solved with a more efficient method. The core idea in their work was that the sequence of operations was construed as the task set, which resonates with our view of task sequences.

The present interpretation of task set may prove useful in developing theories of executive control. Logan and Gordon (2001) proposed a theory of executive control in which a task set is represented at two levels: a task level (a propositional representation derived from task instructions) and a parameter level (a set of control parameters derived from the propositional representation). Task sets are implemented and reconfigured by transmitting control parameters to lower level processing mechanisms (for a related idea, see Broadbent, 1977). Logan and Gordon assumed that "the task-level representation is hierarchical, with the higher level specifying the order in which the tasks occur and the lower level specifying each task separately" (2001, p. 396). This tasklevel representation is comparable to the hierarchical control structure we have proposed and is consistent with some conceptions of motor programs (e.g., Rumelhart \& Norman, 1982). The hierarchical control structure can be accessed to determine the appropriate task to perform on a given trial, and the relevant control parameters can be transmitted to lower level task processes. It may be possible to extend Logan and Gordon's work by specifying the hierarchical control structure in greater detail and implementing the different types of sequence-level processes that act on that structure.

One possible extension would be to integrate Logan and Gordon's (2001) theory of executive control with Anderson and Matessa's (1997; see also Anderson et al., 1998) theory of serial memory. Anderson and Matessa modeled a variety of serial memory phenomena, including the sequence and chunk initiation time effects described earlier, with the adaptive character of thoughtrational (ACT-R; Anderson, 1993) production system. In the ACT-R framework, there are two types of knowledge: declarative and procedural. Declarative knowledge is represented by knowledge units, and procedural knowledge is represented by production 
rules that act on those knowledge units in accordance with currently relevant goals. Anderson and Matessa proposed that a sequence of items can be represented as a hierarchical structure of knowledge units (e.g., see their Figure 2, p. 732). At the bottom level of the hierarchy are individual knowledge units with item identity and position codes. At an intermediate level, these knowledge units may be grouped into larger units representing chunks of items. At the highest level, the chunks are grouped into a single unit representing the entire sequence of items. To access each item in this hierarchical structure, as might be required in serial recall, Anderson and Matessa introduced a series of production rules for retrieving item and chunk information. Two production rules that are highly relevant to the present study are the start-recall and prepare productions (Anderson \& Matessa, 1997, p. 733), which set up a retrieval plan for initiating serial recall. These production rules are akin to the sequence-level processes that are ostensibly required for sequence initiation in our experiments. Slow sequence initiation time would be a corollary of the application of these time-consuming production rules.

Integrating Anderson and Matessa's (1997) production rules for preparing and changing retrieval plans with Logan and Gordon's (2001) architecture for executive control may represent a viable method for modeling sequence initiation, sequence switching, and task switching in the present study and other work (e.g., Logan, 2004; see also Logan, in press). We defer such modeling efforts to the future, however, because our goal in the present study was to uncover some of the empirical phenomena associated with switching tasks in sequences, in an effort to understand more about hierarchical control.

\section{Control}

Control is often conceptualized in terms of a process or set of processes. For example, reconfiguring a task set is thought to involve "executive control processes" such as shifting attention, retrieving goals or task rules, inhibiting an irrelevant task set, or activating a relevant task set (Mayr \& Keele, 2000; Mayr \& Kliegl, 2000; Meiran, 1996; Monsell, 2003; Rubinstein, Meyer, \& Evans, 2001). This process-oriented approach has yielded many insights into the nature of executive control and continues to motivate research on topics such as task switching.

We interpret control in the present study as a relationship between representations and processes at different levels of a hierarchical control structure: Representations at higher levels control processes at lower levels. These higher level representations determine the initiation and functioning of lower level processes, and control is embodied in this determination by representation. Higher level representations can be interpreted as goals to be accomplished by lower level processes (as in ACT-R; Anderson, 1993) or as parameters for programming lower level processes (Logan \& Gordon, 2001; see also Broadbent, 1977). A critical point is that there are no control processes in the typical sense; control is instantiated in the relationship between representations and processes. Applied to our experiments, sequence-level processing associated with sequence initiation and switching does not actively control task-level processing. Instead, hierarchical control arises from the dependence of task-level processes on sequencelevel representations that can be modified by sequence initiation and switching.
We argue that these control relationships are transient. The flow of control between levels in a hierarchy may change depending on the situation, with the "controller" in one situation becoming "controlled" in another situation. This role reversal implies a heterarchical control relationship among cognitive processes: What is designated as "higher" or "lower" in the hierarchy is dynamic rather than fixed (see also Monsell, 1996, pp. 100-102; Rumelhart \& Norman, 1982). This dynamic relationship challenges the familiar distinction between top-down and bottom-up control and is related to the "top-top" control described by Roepstorff and Frith (2004) and foreshadowed by Miller et al. (1960). Furthermore, this control can be explained in terms of basic processes of attention, memory, and perception; we do not have to posit specialized control processes. For example, in the proposed model that integrates the work of Anderson and Matessa (1997) and Logan and Gordon (2001), sequence initiation and switching would be accomplished by memory retrieval. Control arises from the relationship between memory retrieval and task-level processing (which is shaped by the contents of working memory). From this perspective, we are not adopting a reductionist view of control: Describing the basic processes in which control relationships are embodied does not reduce control to the operation of those processes or eliminate the concept of control. We may be able to specify the processes that generate the representations that control other processes, but the control relationship remains.

The dynamic hierarchical control relationship outlined above represents another way of thinking about the flexibility that is expressed in most definitions of control. An important implication is that it may not be possible to identify fixed sources of control in behavior. If control is embodied in a hierarchical relationship between different representations and processes, then there is unlikely to be a single locus that remains unchanged across different situations. This implies that the concept of control can be understood in some situations without recourse to an omniscient homunculus. Instead, understanding the control relationship may be sufficient for explaining control. In this sense, we may have taken another step toward what some researchers view as a central goal of cognitive science: "banishing the control homunculus" (Monsell \& Driver, 2000a, p. 3).

\section{Conclusion}

Complex patterns of behavior often unfold according to plans. In the present study, we found evidence of hierarchical control of cognitive processes in experiments involving the performance of explicit, memorized task sequences. The effects of sequence initiation and sequence switching on switch costs-especially switch costs at the first serial position across sequences-highlighted the relationship between the sequence and task levels, suggesting that task switching occurred within a hierarchical control structure. Further research on the nature of hierarchical control in cognition is important for understanding how plans such as recipes can guide our behavior. A research approach that tackles the issue from a variety of angles - ranging from motor programs to serial memory to task sets-may represent a recipe for success.

\section{References}

Allport, A., Styles, E. A., \& Hsieh, S. (1994). Shifting intentional set: Exploring the dynamic control of tasks. In C. Umiltà \& M. Moscovitch 
(Eds.), Attention and performance XV: Conscious and nonconscious information processing (pp. 421-452). Cambridge, MA: MIT Press.

Allport, A., \& Wylie, G. (2000). Task switching, stimulus-response bindings, and negative priming. In S. Monsell \& J. Driver (Eds.), Control of cognitive processes: Attention and performance XVIII (pp. 35-70). Cambridge, MA: MIT Press.

Altmann, E. M. (2003). Task switching and the pied homunculus: Where are we being led? Trends in Cognitive Sciences, 7, 340-341.

Altmann, E. M. (2004). The preparation effect in task switching: Carryover of SOA. Memory \& Cognition, 32, 153-163.

Anderson, J. R. (1993). Rules of the mind. Hillsdale, NJ: Erlbaum.

Anderson, J. R., Bothell, D., Lebiere, C., \& Matessa, M. (1998). An integrated theory of list memory. Journal of Memory and Language, 38, 341-380.

Anderson, J. R., \& Matessa, M. (1997). A production system theory of serial memory. Psychological Review, 104, 728-748.

Botvinick, M., \& Plaut, D. C. (2004). Doing without schema hierarchies: A recurrent connectionist approach to normal and impaired routine sequential action. Psychological Review, 111, 395-429.

Bower, G. H. (1970). Organizational factors in memory. Cognitive Psychology, 1, 18-46.

Bower, G. H., \& Winzenz, D. (1969). Group structure, coding, and memory for digit series. Journal of Experimental Psychology, 80(2, Pt. 2), $1-17$.

Broadbent, D. E. (1977). Levels, hierarchies, and the locus of control. Quarterly Journal of Experimental Psychology, 29, 181-201.

Cohen, G. (2000). Hierarchical models in cognition: Do they have psychological reality? European Journal of Cognitive Psychology, 12, $1-36$.

Cooper, R., \& Shallice, T. (2000). Contention scheduling and the control of routine activities. Cognitive Neuropsychology, 17, 297-338.

Cooper, R. P., \& Shallice, T. (in press). Hierarchical schemas and goals in the control of sequential behavior. Psychological Review.

Dashiell, J. F. (1940). A neglected fourth dimension to psychological research. Psychological Review, 47, 289-305.

De Jong, R. (1995). The role of preparation in overlapping-task performance. Quarterly Journal of Experimental Psychology, 48A, 2-25.

Dixon, P. (1987). The structure of mental plans for following directions. Journal of Experimental Psychology: Learning, Memory, and Cognition, 13, 18-26.

Dreisbach, G., Haider, H., \& Kluwe, R. H. (2002). Preparatory processes in the task-switching paradigm: Evidence from the use of probability cues. Journal of Experimental Psychology: Learning, Memory, and Cognition, 28, 468-483.

Gibson, J. J. (1941). A critical review of the concept of set in contemporary experimental psychology. Psychological Bulletin, 38, 781-817.

Gordon, P. C., \& Meyer, D. E. (1987). Control of serial order in rapidly spoken syllable sequences. Journal of Memory and Language, 26, 300321

Gotler, A., Meiran, N., \& Tzelgov, J. (2003). Nonintentional task set activation: Evidence from implicit task sequence learning. Psychonomic Bulletin \& Review, 10, 890-896.

Henry, F. M., \& Rogers, D. E. (1960). Increased response latency for complicated movements and a "memory drum" theory of neuromotor reaction. Research Quarterly, 31, 448-458.

Heuer, H., Schmidtke, V., \& Kleinsorge, T. (2001). Implicit learning of sequences of tasks. Journal of Experimental Psychology: Learning, Memory, and Cognition, 27, 967-983.

Jersild, A. T. (1927). Mental set and shift. Archives of Psychology, 14(Whole No. 89)

Johnson, N. F. (1970). The role of chunking and organization in the process of recall. In G. H. Bower (Ed.), The psychology of learning and motivation (Vol. 4, pp. 171-247). New York: Academic Press.

Kahana, M. J., \& Jacobs, J. (2000). Interresponse times in serial recall:
Effects of intraserial repetition. Journal of Experimental Psychology: Learning, Memory, and Cognition, 26, 1188-1197.

Keele, S. W., Cohen, A., \& Ivry, R. (1990). Motor programs: Concepts and issues. In M. Jeannerod (Ed.), Attention and performance XIII: Motor representation and control (pp. 77-110). Hillsdale, NJ: Erlbaum.

Keele, S. W., \& Summers, J. J. (1976). The structure of motor programs. In G. E. Stelmach (Ed.), Motor control: Issues and trends (pp. 109142). New York: Academic Press.

Klapp, S. T., Anderson, W. G., \& Berrian, R. W. (1973). Implicit speech in reading, reconsidered. Journal of Experimental Psychology, 100, $368-374$.

Kleinsorge, T., \& Heuer, H. (1999). Hierarchical switching in a multidimensional task space. Psychological Research, 62, 300-312.

Kleinsorge, T., Heuer, H., \& Schmidtke, V. (2004). Assembling a task space: Global determination of local shift costs. Psychological Research $68,31-40$.

Kleinsorge, T., Schmidtke, V., Gajewski, P. D., \& Heuer, H. (2003). The futility of explicit knowledge of a sequence of tasks. European Journal of Cognitive Psychology, 15, 455-469.

Koch, I. (2001). Automatic and intentional activation of task sets. Journal of Experimental Psychology: Learning, Memory, and Cognition, 27 $1474-1486$

Koch, I. (2003). The role of external cues for endogenous advance reconfiguration in task switching. Psychonomic Bulletin \& Review, 10, 488 492.

Koch, I. (2005). Sequential task predictability in task switching. Psychonomic Bulletin \& Review, 12, 107-112.

Koch, I., \& Hoffmann, J. (2000). Patterns, chunks, and hierarchies in serial reaction-time tasks. Psychological Research, 63, 22-35.

Koch, I., Philipp, A. M., \& Gade, M. (2006). Chunking in task sequences modulates task inhibition. Psychological Science, 17, 346-350.

Lashley, K. S. (1951). The problem of serial order in behavior. In L. A. Jeffress (Ed.), Cerebral mechanisms in behavior (pp. 112-136). New York: Wiley.

Lien, M.-C., \& Ruthruff, E. (2004). Task switching in a hierarchical task structure: Evidence for the fragility of the task repetition benefit. Journal of Experimental Psychology: Learning, Memory, and Cognition, 30, 697-713.

Logan, G. D. (1985). Executive control of thought and action. Acta Psychologica, 60, 193-210.

Logan, G. D. (2003). Executive control of thought and action: In search of the wild homunculus. Current Directions in Psychological Science, 12 $45-48$.

Logan, G. D. (2004). Working memory, task switching, and executive control in the task span procedure. Journal of Experimental Psychology: General, 133, 218-236.

Logan, G. D. (2006). Out with the old, in with the new: More valid measures of switch cost and retrieval time in the task span procedure Psychonomic Bulletin \& Review, 13, 139-144.

Logan, G. D. (in press). What it costs to implement a plan: Plan-level and task-level contributions to switch costs. Memory \& Cognition.

Logan, G. D., \& Gordon, R. D. (2001). Executive control of visual attention in dual-task situations. Psychological Review, 108, 393-434.

Luchins, A. S., \& Luchins, E. H. (1950). New experimental attempts at preventing mechanization in problem solving. Journal of General Psychology, 42, 279-297.

Luria, R., \& Meiran, N. (2003). Online order control in the psychological refractory period paradigm. Journal of Experimental Psychology: $\mathrm{Hu}$ man Perception and Performance, 29, 556-574.

Mayr, U. (2003). Towards principles of executive control: How mental sets are selected. In R. H. Kluwe, G. Lüer, \& F. Rösler (Eds.), Principles of learning and memory (Vol. 1, pp. 223-241). Basel, Switzerland: Birkhäuser.

Mayr, U., \& Keele, S. W. (2000). Changing internal constraints on action: 
The role of backward inhibition. Journal of Experimental Psychology: General, 129, 4-26.

Mayr, U., \& Kliegl, R. (2000). Task-set switching and long-term memory retrieval. Journal of Experimental Psychology: Learning, Memory, and Cognition, 26, 1124-1140.

McClelland, J. L. (1979). On the time relations of mental processes: An examination of systems of processes in cascade. Psychological Review, 86, 287-330.

Meiran, N. (1996). Reconfiguration of processing mode prior to task performance. Journal of Experimental Psychology: Learning, Memory, and Cognition, 22, 1423-1442.

Meiran, N., Chorev, Z., \& Sapir, A. (2000). Component processes in task switching. Cognitive Psychology, 41, 211-253.

Miller, G. A., Galanter, E., \& Pribram, K. H. (1960). Plans and the structure of behavior. New York: Holt, Rinehart \& Winston.

Monsell, S. (1996). Control of mental processes. In V. Bruce (Ed.), Unsolved mysteries of the mind (pp. 93-148). Hove, England: Erlbaum.

Monsell, S. (2003). Task switching. Trends in Cognitive Sciences, 7 , $134-140$.

Monsell, S., \& Driver, J. (2000a). Banishing the control homunculus. In S. Monsell \& J. Driver (Eds.), Control of cognitive processes: Attention and performance XVIII (pp. 3-32). Cambridge, MA: MIT Press.

Monsell, S., \& Driver, J. (Eds.). (2000b). Control of cognitive processes: Attention and performance XVIII. Cambridge, MA: MIT Press.

Monsell, S., \& Mizon, G. A. (2006). Can the task-cuing paradigm measure an endogenous task-set reconfiguration process? Journal of Experimental Psychology: Human Perception and Performance, 32, 493-516.

Norman, D. A., \& Shallice, T. (1986). Attention to action: Willed and automatic control of behavior. In R. J. Davidson, G. E. Schwartz, \& D. Shapiro (Eds.), Consciousness and self-regulation (Vol. 4, pp. 1-18). New York: Plenum Press.

Pashler, H. (1984). Processing stages in overlapping tasks: Evidence for a central bottleneck. Journal of Experimental Psychology: Human Perception and Performance, 10, 358-377.

Povel, D.-J., \& Collard, R. (1982). Structural factors in patterned finger tapping. Acta Psychologica, 52, 107-123.

Psychology Software Tools. (2002). E-Prime (Version 1.1) [Computer software]. Pittsburgh, PA: Author.

Restle, F., \& Burnside, B. L. (1972). Tracking of serial patterns. Journal of Experimental Psychology, 95, 299-307.

Roepstorff, A., \& Frith, C. (2004). What's at the top in the top-down control of action? Script-sharing and "top-top" control of action in cognitive experiments. Psychological Research, 68, 189-198.

Rogers, R. D., \& Monsell, S. (1995). Costs of a predictable switch between simple cognitive tasks. Journal of Experimental Psychology: General, 124, 207-231.

Rosenbaum, D. A. (1984). The planning and control of movements. In J. R. Anderson \& S. M. Kosslyn (Eds.), Tutorials in learning and memory: Essays in honor of Gordon Bower (pp. 219-233). San Francisco: Freeman.

Rosenbaum, D. A. (1987). Hierarchical organization of motor programs. In
S. Wise (Ed.), Neural and behavioral approaches to higher brain functions (pp. 45-66). New York: Wiley.

Rosenbaum, D. A., Inhoff, A. W., \& Gordon, A. M. (1984). Choosing between movement sequences: A hierarchical editor model. Journal of Experimental Psychology: General, 113, 372-393.

Rosenbaum, D. A., Kenny, S. B., \& Derr, M. A. (1983). Hierarchical control of rapid movement sequences. Journal of Experimental Psychology: Human Perception and Performance, 9, 86-102.

Rubinstein, J. S., Meyer, D. E., \& Evans, J. E. (2001). Executive control of cognitive processes in task switching. Journal of Experimental Psychology: Human Perception and Performance, 27, 763-797.

Rumelhart, D. E., \& Norman, D. A. (1982). Simulating a skilled typist: A study of skilled cognitive-motor performance. Cognitive Science, 6 , $1-36$.

Ruthruff, E., Remington, R. W., \& Johnston, J. C. (2001). Switching between simple cognitive tasks: The interaction of top-down and bottom-up factors. Journal of Experimental Psychology: Human Perception and Performance, 27, 1404-1419.

Schneider, D. W., \& Logan, G. D. (2005). Modeling task switching without switching tasks: A short-term priming account of explicitly cued performance. Journal of Experimental Psychology: General, 134, 343-367.

Schneider, D. W., \& Logan, G. D. (2006). Priming cue encoding by manipulating transition frequency in explicitly cued task switching. Psychonomic Bulletin \& Review, 13, 145-151.

Schweickert, R. (1978). A critical path generalization of the additive factor method: Analysis of a Stroop task. Journal of Mathematical Psychology, $18,105-139$.

Schweickert, R., \& Townsend, J. T. (1989). A trichotomy: Interactions of factors prolonging sequential and concurrent processes in stochastic discrete mental (PERT) networks. Journal of Mathematical Psychology, $33,328-347$

Simon, H. A. (1972). Complexity and the representation of patterned sequences of symbols. Psychological Review, 79, 369-382.

Sohn, M.-H., \& Anderson, J. R. (2001). Task preparation and task repetition: Two-component model of task switching. Journal of Experimental Psychology: General, 130, 764-778.

Sohn, M.-H., \& Carlson, R. A. (2000). Effects of repetition and foreknowledge in task-set reconfiguration. Journal of Experimental Psychology: Learning, Memory, and Cognition, 26, 1445-1460.

Sternberg, S., Monsell, S., Knoll, R. L., \& Wright, C. E. (1978). The latency and duration of rapid movement sequences: Comparisons of speech and typewriting. In G. E. Stelmach (Ed.), Information processing in motor control and learning (pp. 117-152). New York: Academic Press.

Waszak, F., Hommel, B., \& Allport, A. (2003). Task-switching and longterm priming: Role of episodic stimulus-task bindings in task-shift costs. Cognitive Psychology, 46, 361-413.

Received May 24, 2005 Revision received April 19, 2006 Accepted April 28, 2006 